Supporting Information

\title{
The role of nitrogen donors in zinc catalysts for lactide ring opening polymerization
}

Tannaz Ebrahimi, ${ }^{a, b \ddagger}$ Emiliya Mamleeva, ${ }^{\text {ał }}$ Insun $\mathrm{Yu}^{\mathrm{a}},{ }^{\ddagger}{ }^{\ddagger}$ Savvas G. Hatzikiriakos, ${ }^{b}$ and Parisa Mehrkhodavandi ${ }^{\text {a* }}$

${ }^{a}$ Department of Chemistry, University of British Columbia, 2036 Main Mall, Vancouver, British Columbia. ${ }^{b}$ Department of Chemical and Biological Engineering, University of British Columbia, 236o East Mall, Vancouver, British Columbia, Canada.

Fax: 604-822-2847

Email:mehr@chem.ubc.ca

http://www.chem.ubc.ca/personnel/faculty/mehr/index.shtml

\section{Contents}

A. Characterization of compounds 1-7 in solution by ${ }^{1} \mathrm{H}$ NMR and ${ }^{13} \mathrm{C}\left\{{ }^{1} \mathrm{H}\right\} \mathrm{NMR}$............ 2

B. Characterization of compounds 2-7 in the solid state ............................................ 9

C. Characterization of compounds $1-6$ in solution by PFGSE NMR ................................. 13

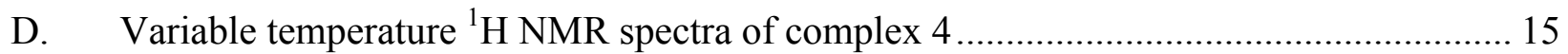

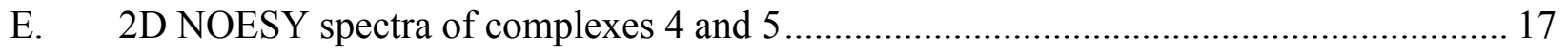

F. Ring opening polymerization of rac-lactide catalyzed by complexes $1-3 \ldots \ldots \ldots \ldots \ldots \ldots \ldots . . . . . . . .22$

G. MALDI-TOF mass and ${ }^{1}$ H NMR spectra of PLA produced by complexes 4-6 .............. 24

H. Homonuclear decoupled ${ }^{1}$ H NMR spectra................................................................. 29

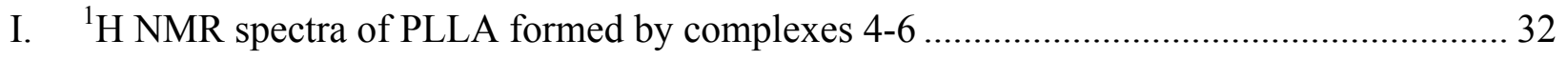

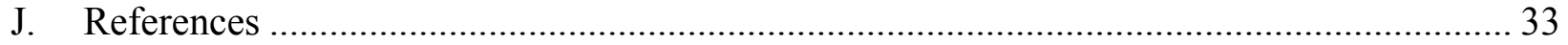


A. Characterization of compounds 1-7 in solution by ${ }^{1} \mathrm{H}$ NMR and ${ }^{13} \mathrm{C}\left\{{ }^{1} \mathrm{H}\right\}$ NMR

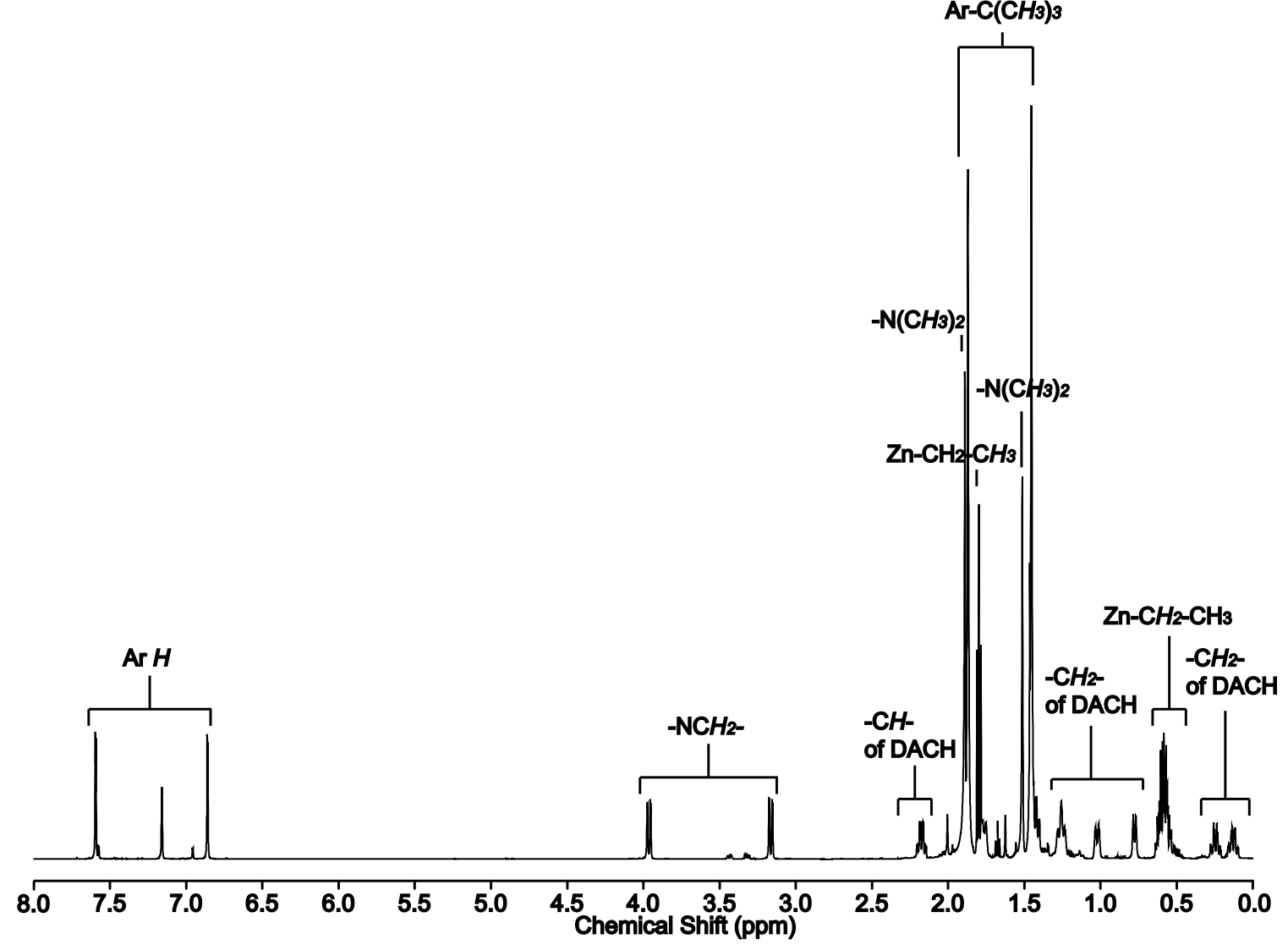

Figure S1. ${ }^{1} \mathrm{H}$ NMR spectrum $\left(600 \mathrm{MHz}, 25^{\circ} \mathrm{C}, \mathrm{C}_{6} \mathrm{D}_{6}\right)$ of complex $1( \pm)-\left(\mathrm{NN}_{\mathrm{H}} \mathrm{O}_{t-\mathrm{Bu}}\right) \mathrm{Zn}\left(\mathrm{CH}_{2} \mathrm{CH}_{3}\right)$.

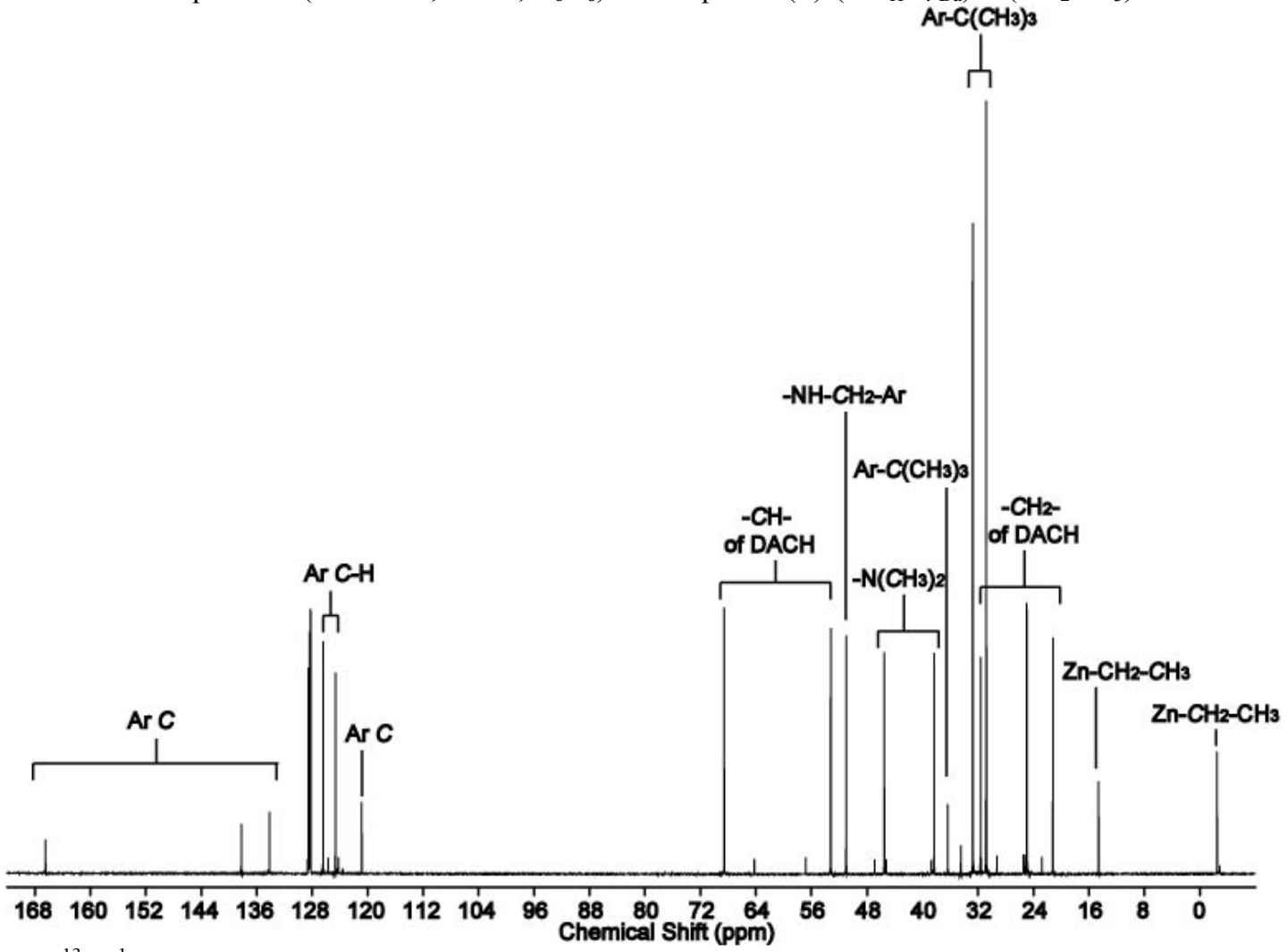

Figure S2. ${ }^{13} \mathrm{C}\left\{{ }^{1} \mathrm{H}\right\}$ NMR spectrum $\left(600 \mathrm{MHz}, 25{ }^{\circ} \mathrm{C}, \mathrm{C}_{6} \mathrm{D}_{6}\right)$ of complex $\mathbf{1}( \pm)-\left(\mathrm{NN}_{\mathrm{H}} \mathrm{O}_{t-\mathrm{Bu}}\right) \mathrm{Zn}\left(\mathrm{CH}_{2} \mathrm{CH}_{3}\right)$. 


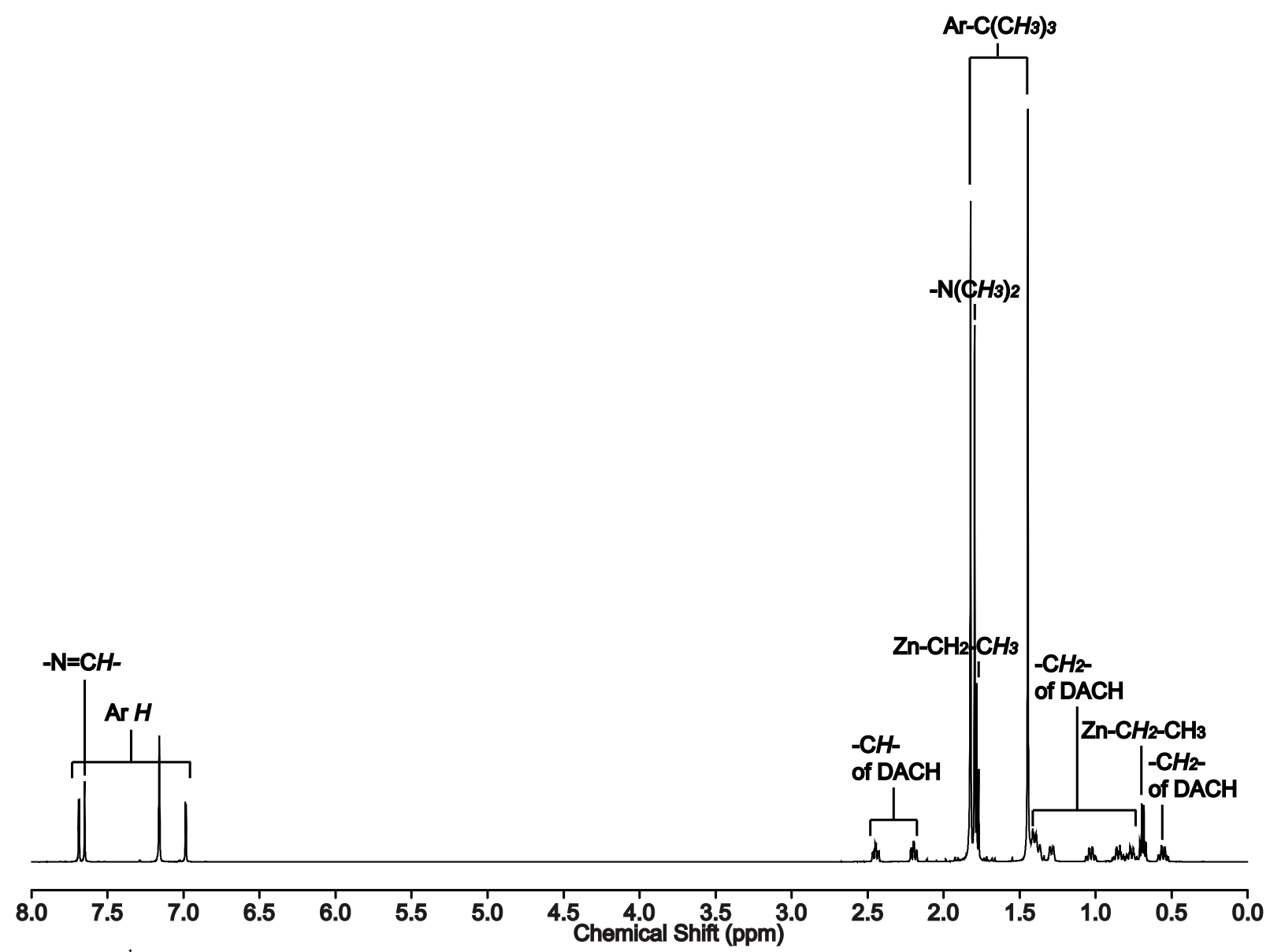

Figure S3. ${ }^{1} \mathrm{H}$ NMR spectrum $\left(600 \mathrm{MHz}, 25^{\circ} \mathrm{C}, \mathrm{C}_{6} \mathrm{D}_{6}\right)$ of complex $2( \pm)-\left(\mathrm{NNO}_{t-\mathrm{Bu}}\right) \mathrm{Zn}\left(\mathrm{CH}_{2} \mathrm{CH}_{3}\right)$.

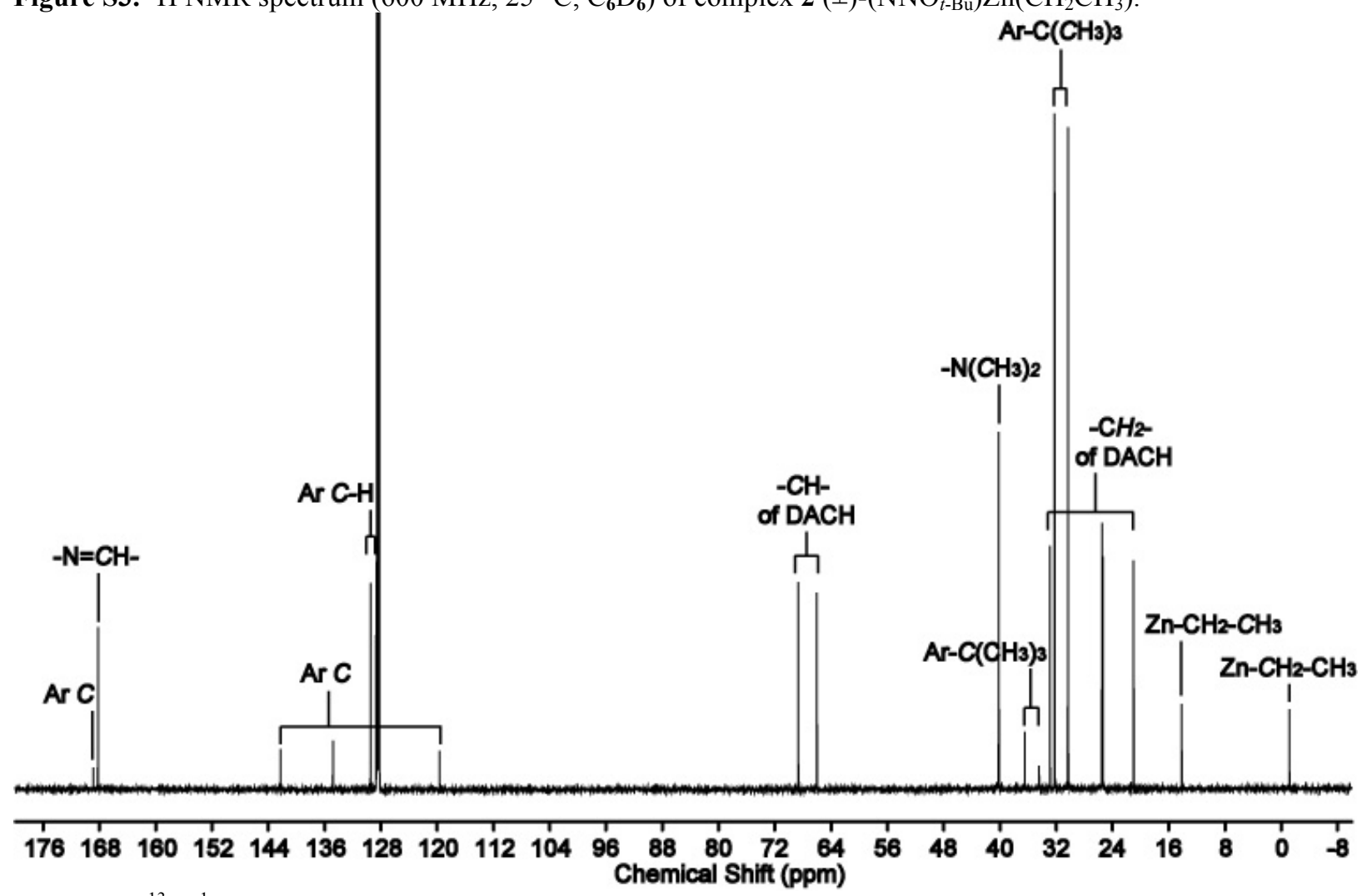

Figure S4. ${ }^{13} \mathrm{C}\left\{{ }^{1} \mathrm{H}\right\}$ NMR spectrum $\left(600 \mathrm{MHz}, 25^{\circ} \mathrm{C}, \mathrm{C}_{6} \mathrm{D}_{6}\right)$ of complex $2( \pm)-\left(\mathrm{NNO}_{t-\mathrm{Bu}}\right) \mathrm{Zn}\left(\mathrm{CH}_{2} \mathrm{CH}_{3}\right)$. 


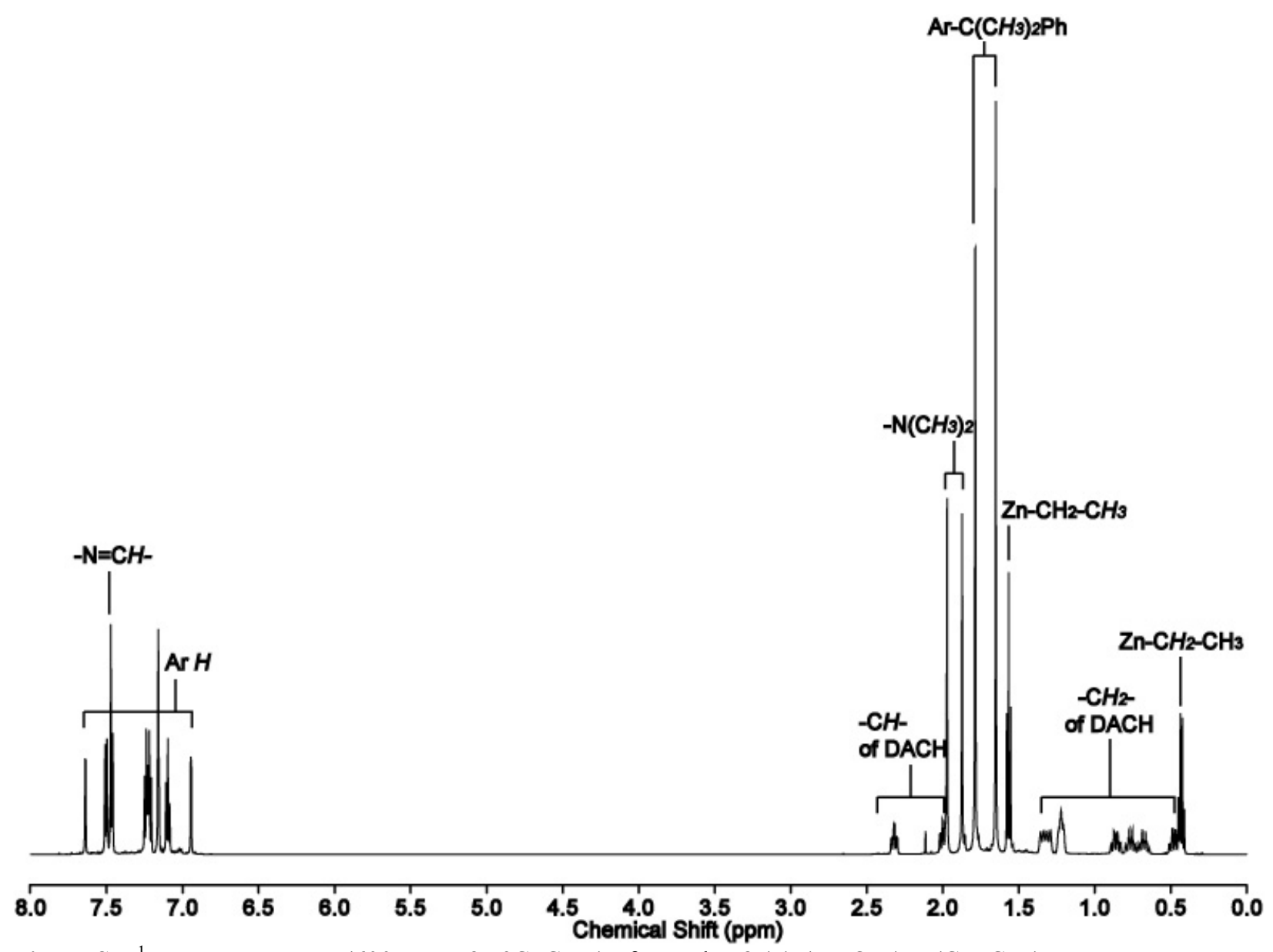

Figure S5. ${ }^{1} \mathrm{H}$ NMR spectrum $\left(600 \mathrm{MHz}, 25^{\circ} \mathrm{C}, \mathrm{C}_{6} \mathrm{D}_{6}\right)$ of complex $3( \pm)-\left(\mathrm{NNO}_{\mathrm{Cm}}\right) \mathrm{Zn}\left(\mathrm{CH}_{2} \mathrm{CH}_{3}\right)$.

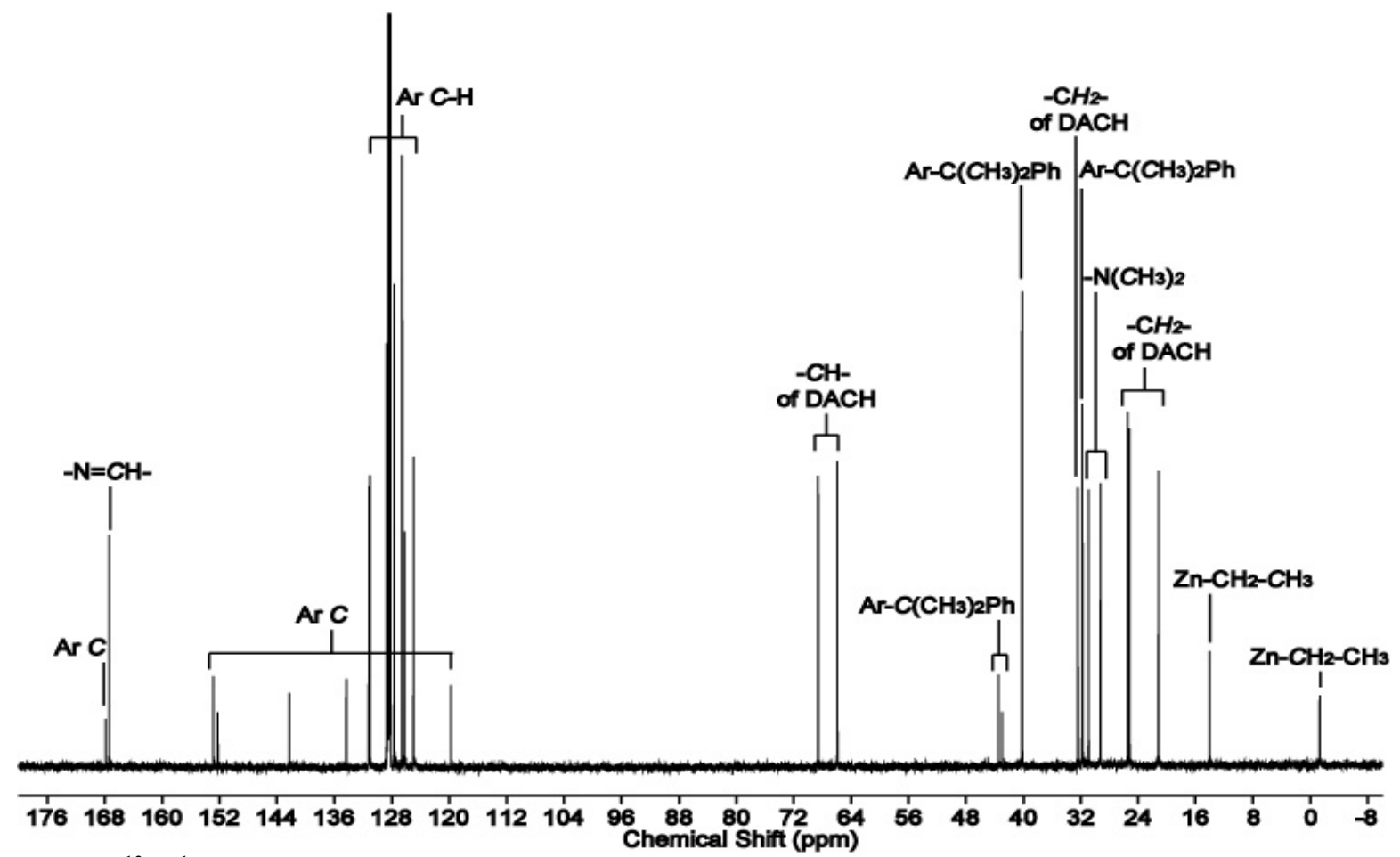

Figure S6. ${ }^{13} \mathrm{C}\left\{{ }^{1} \mathrm{H}\right\}$ NMR spectrum $\left(600 \mathrm{MHz}, 25^{\circ} \mathrm{C}, \mathrm{C}_{6} \mathrm{D}_{6}\right)$ of complex $3( \pm)-\left(\mathrm{NNO}_{\mathrm{Cm}}\right) \mathrm{Zn}\left(\mathrm{CH}_{2} \mathrm{CH}_{3}\right)$. 


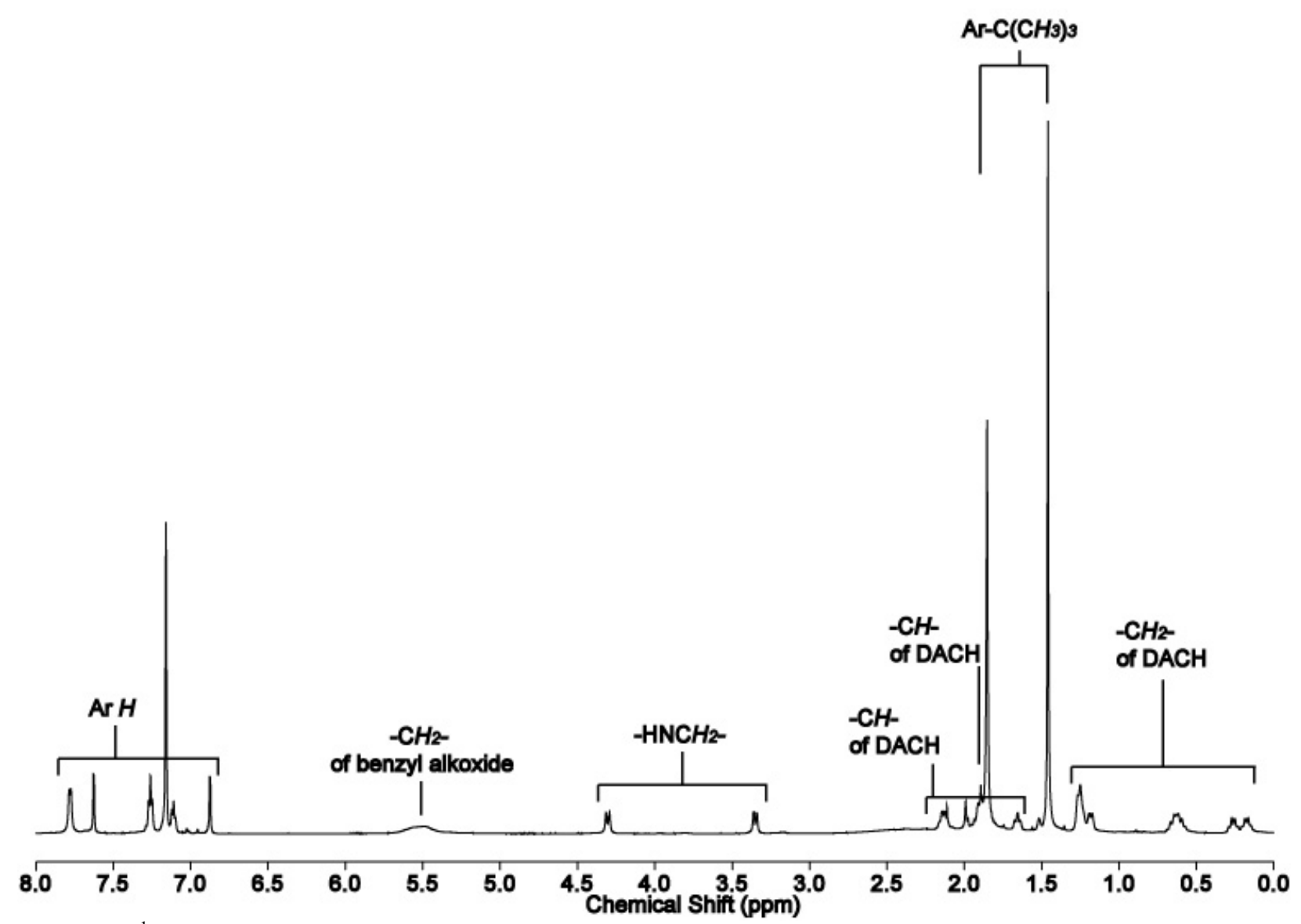

Figure S7. ${ }^{1} \mathrm{H}$ NMR spectrum $\left(600 \mathrm{MHz}, 25^{\circ} \mathrm{C}, \mathrm{C}_{6} \mathrm{D}_{6}\right)$ of complex $4( \pm)-\left[\left(\mathrm{NN}_{\mathrm{H}} \mathrm{O}_{t-\mathrm{Bu}}\right) \mathrm{Zn}\left(\mathrm{OCH}_{2} \mathrm{Ph}\right)\right]_{2}$.

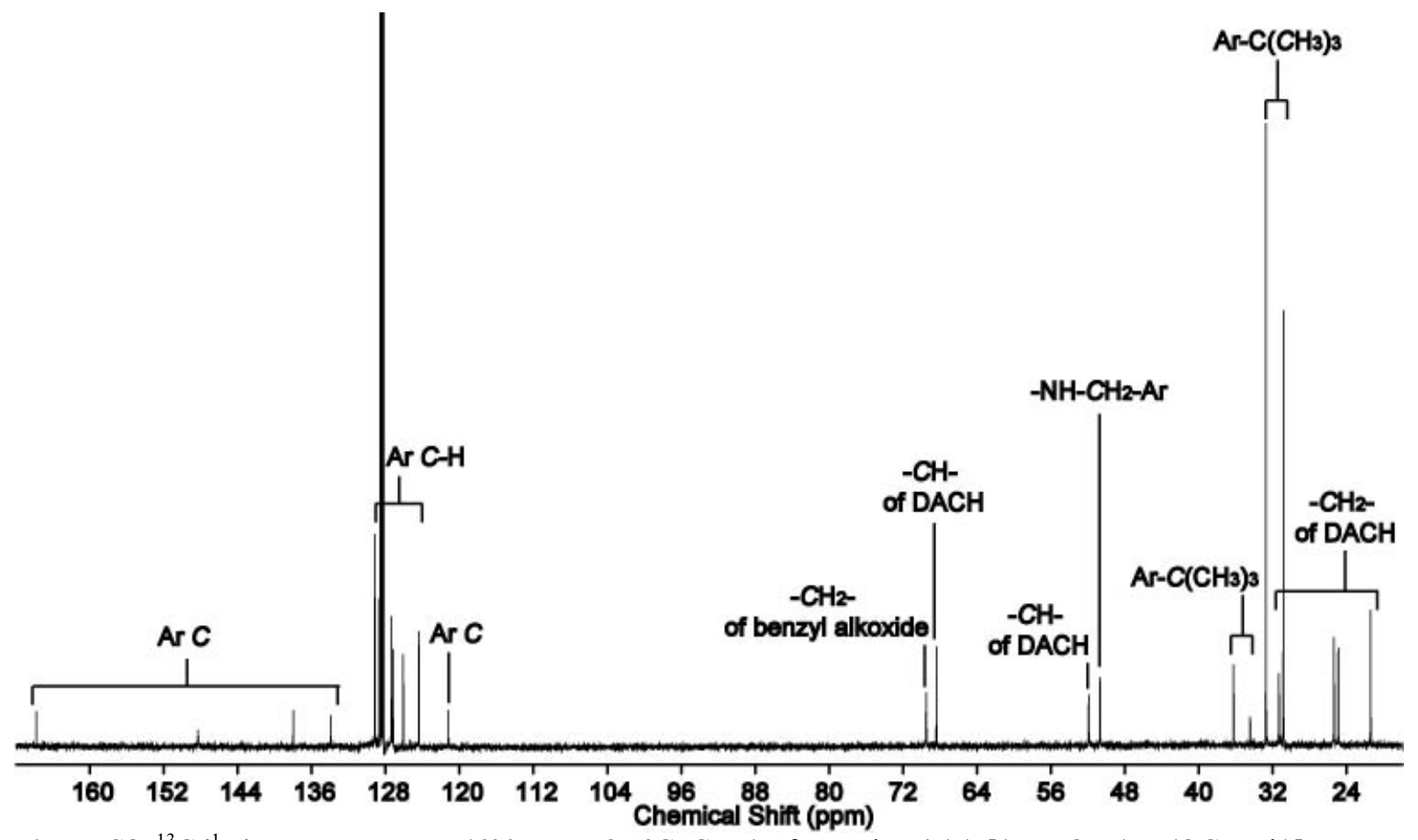

Figure S8. ${ }^{13} \mathrm{C}\left\{{ }^{1} \mathrm{H}\right\}$ NMR spectrum $\left(600 \mathrm{MHz}, 25{ }^{\circ} \mathrm{C}, \mathrm{C}_{6} \mathrm{D}_{6}\right)$ of complex $4( \pm)-\left[\left(\mathrm{NN}_{\mathrm{H}} \mathrm{O} t \mathrm{Bu}\right) \mathrm{Zn}\left(\mathrm{OCH}_{2} \mathrm{Ph}\right)\right]_{2}$. 


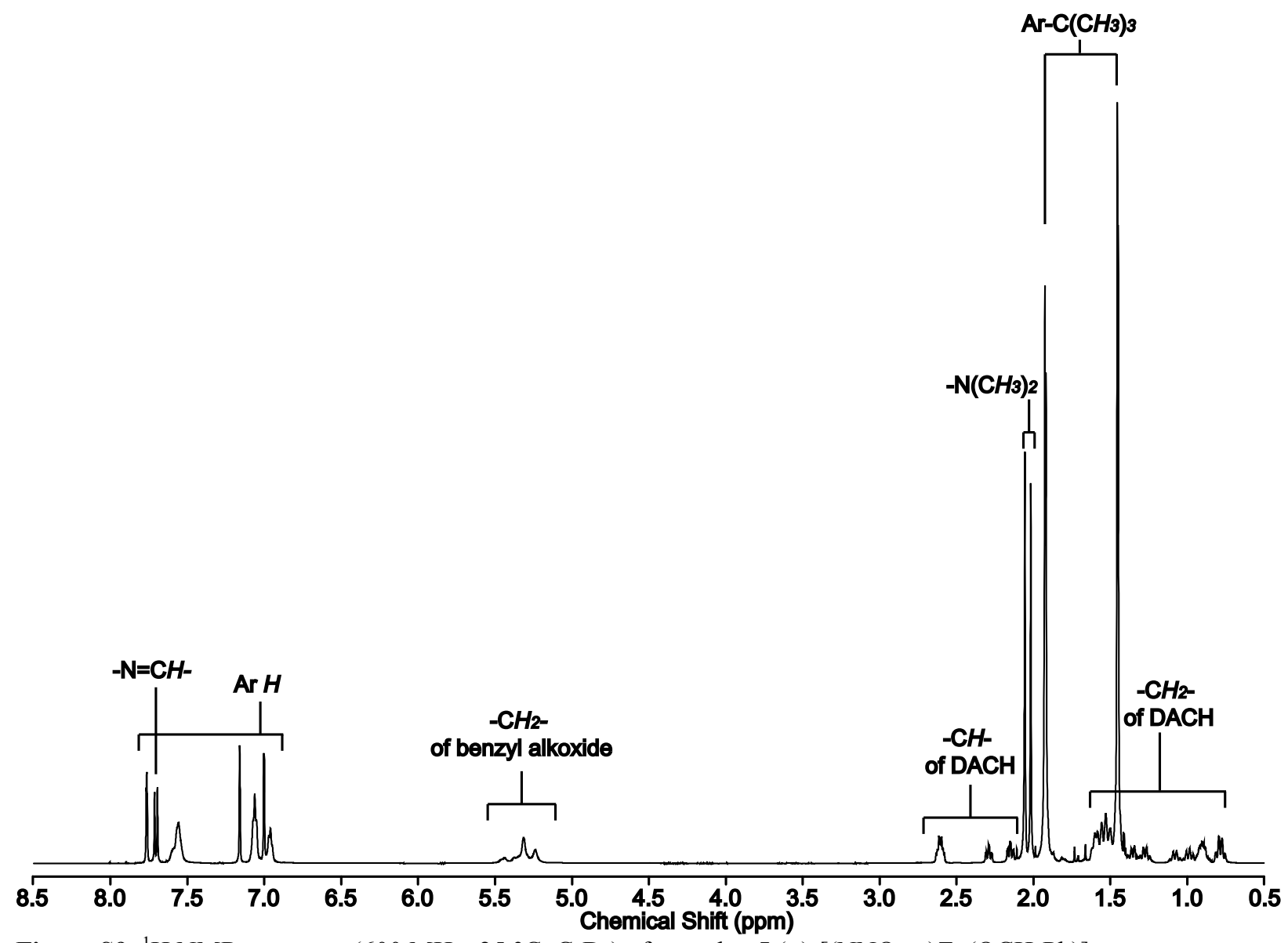

Figure S9. ${ }^{1} \mathrm{H}$ NMR spectrum $\left(600 \mathrm{MHz}, 25^{\circ} \mathrm{C}, \mathrm{C}_{6} \mathrm{D}_{\mathbf{6}}\right)$ of complex $5( \pm)-\left[\left(\mathrm{NNO}_{t-\mathrm{Bu}}\right) \mathrm{Zn}\left(\mathrm{OCH}_{2} \mathrm{Ph}\right)\right]_{2}$.

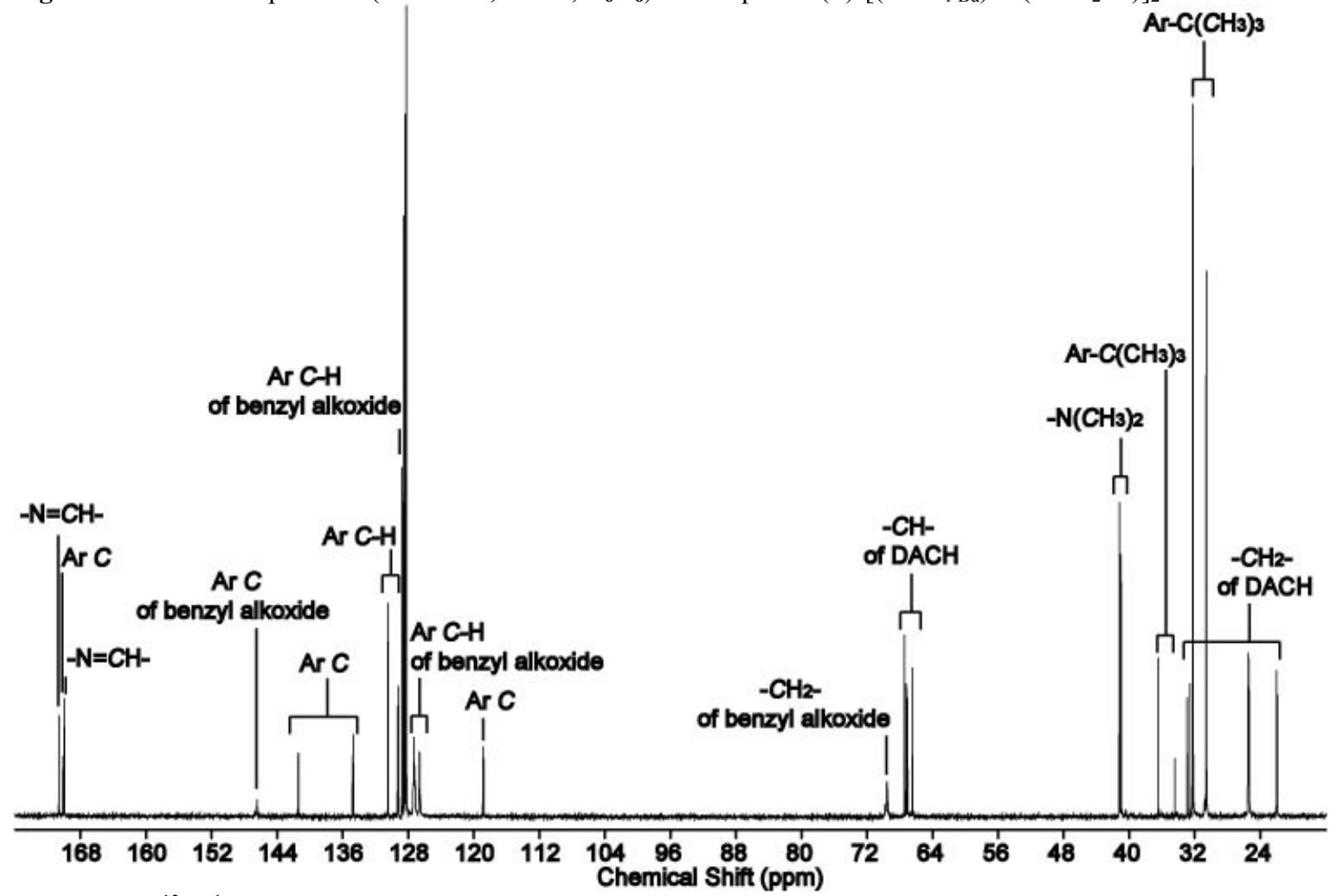

Figure S10. ${ }^{13} \mathrm{C}\left\{{ }^{1} \mathrm{H}\right\}$ NMR spectrum $\left(600 \mathrm{MHz}, 25^{\circ} \mathrm{C}, \mathrm{C}_{6} \mathrm{D}_{6}\right)$ of complex $5( \pm)-\left[\left(\mathrm{NNO}_{t-\mathrm{Bu}}\right) \mathrm{Zn}\left(\mathrm{OCH}_{2} \mathrm{Ph}\right)\right]_{2}$. 


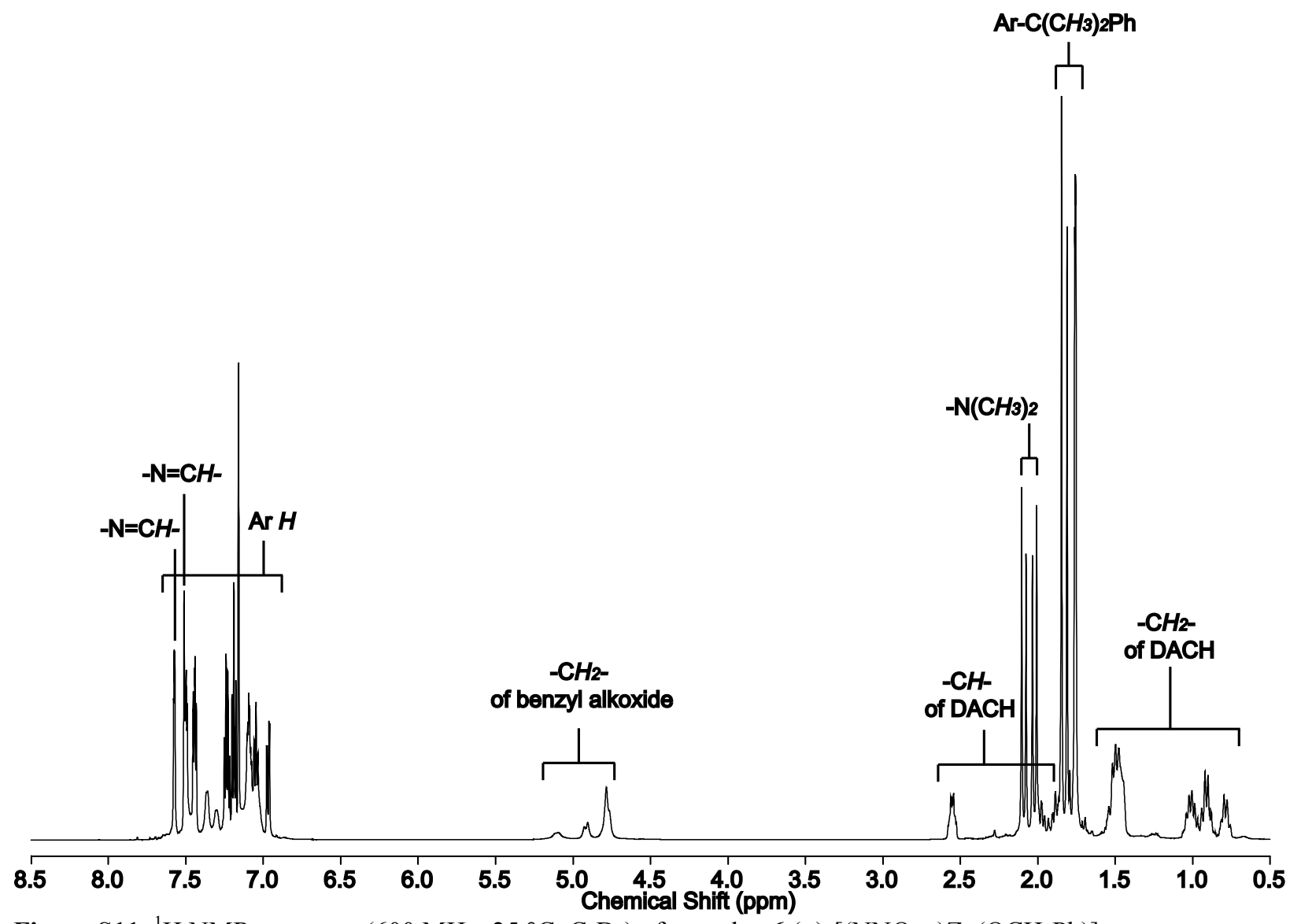

Figure S11. ${ }^{1} \mathrm{H}$ NMR spectrum $\left(600 \mathrm{MHz}, 25^{\circ} \mathrm{C}, \mathrm{C}_{6} \mathrm{D}_{6}\right)$ of complex $6( \pm)-\left[\left(\mathrm{NNO}_{\mathrm{Cm}}\right) \mathrm{Zn}\left(\mathrm{OCH}_{2} \mathrm{Ph}\right)\right]_{2}$.

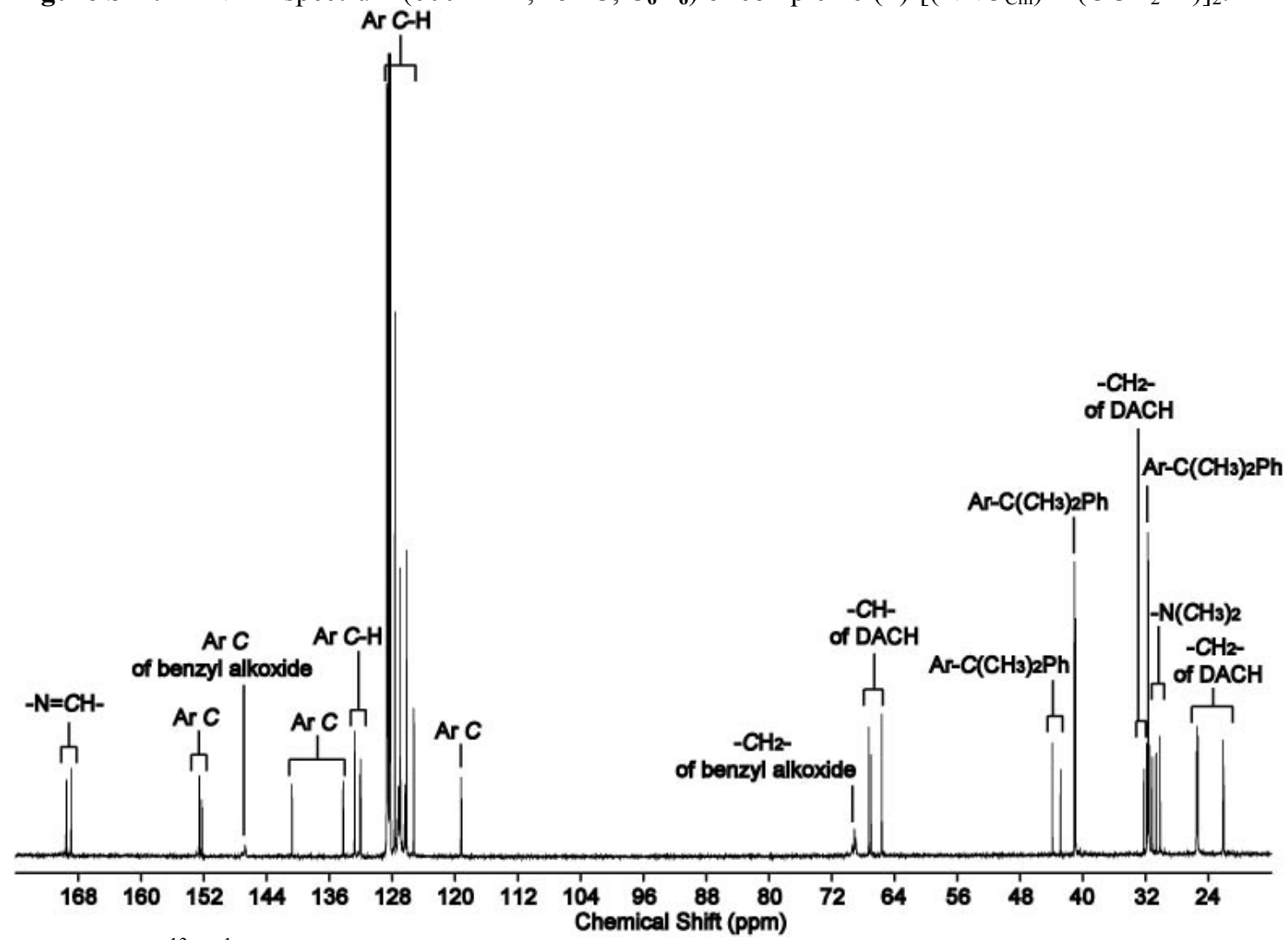

Figure S12. ${ }^{13} \mathrm{C}\left\{{ }^{1} \mathrm{H}\right\}$ NMR spectrum $\left(600 \mathrm{MHz}, 25{ }^{\circ} \mathrm{C}, \mathrm{C}_{6} \mathrm{D}_{6}\right)$ of complex $6( \pm)-\left[\left(\mathrm{NNO}_{\mathrm{Cm}}\right) \mathrm{Zn}\left(\mathrm{OCH}_{2} \mathrm{Ph}\right)\right]_{2}$. 


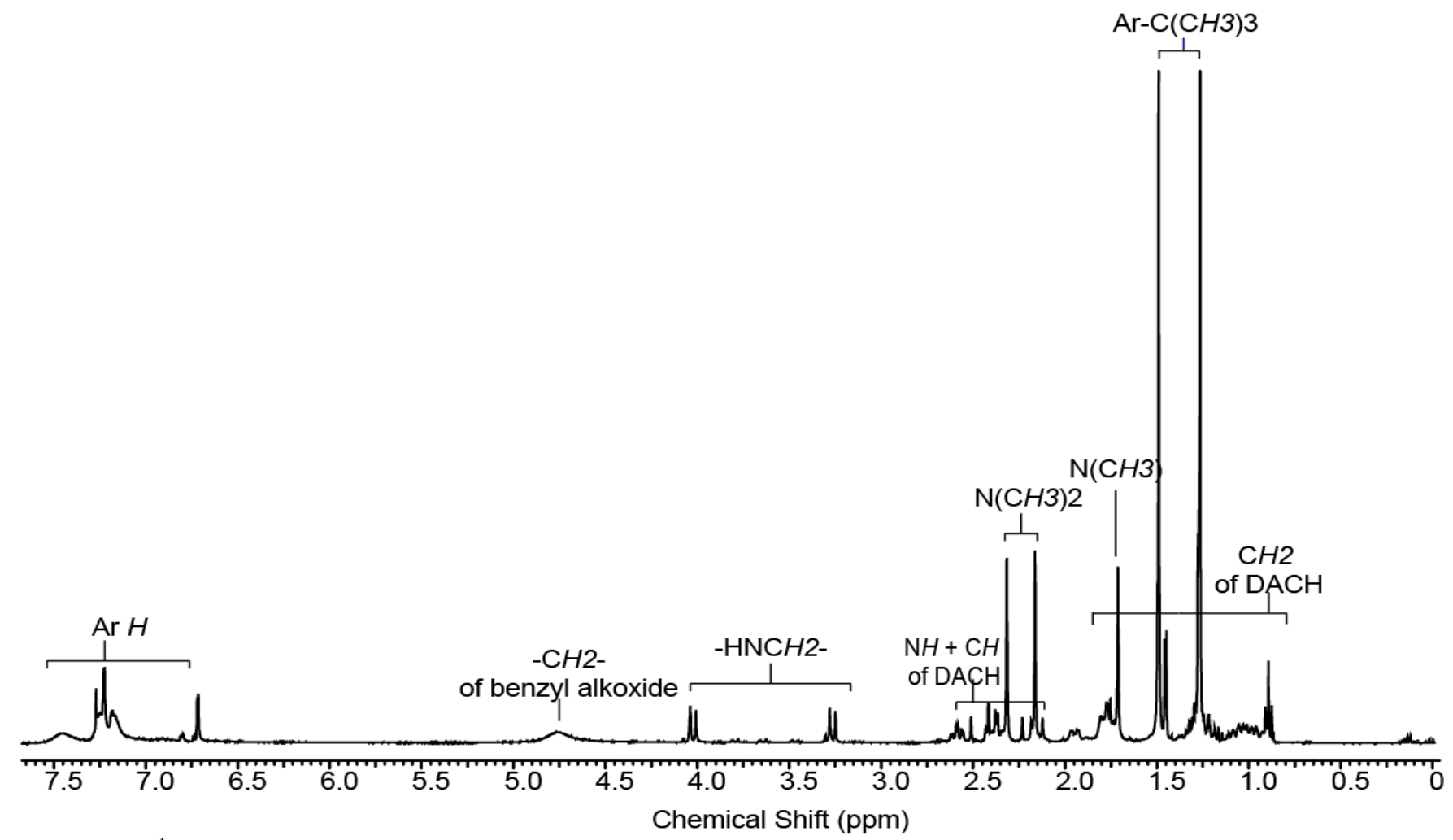

Figure S13. ${ }^{1} \mathrm{H}$ NMR spectrum $\left(600 \mathrm{MHz}, 25^{\circ} \mathrm{C}, \mathrm{CDCl}_{3}\right)$ of complex $7( \pm)-\left(\mathrm{NN}_{\mathrm{Me}} \mathrm{O}_{\mathrm{tBu}}\right) \mathrm{Zn}\left(\mathrm{OCH}_{2} \mathrm{Ph}\right)$.

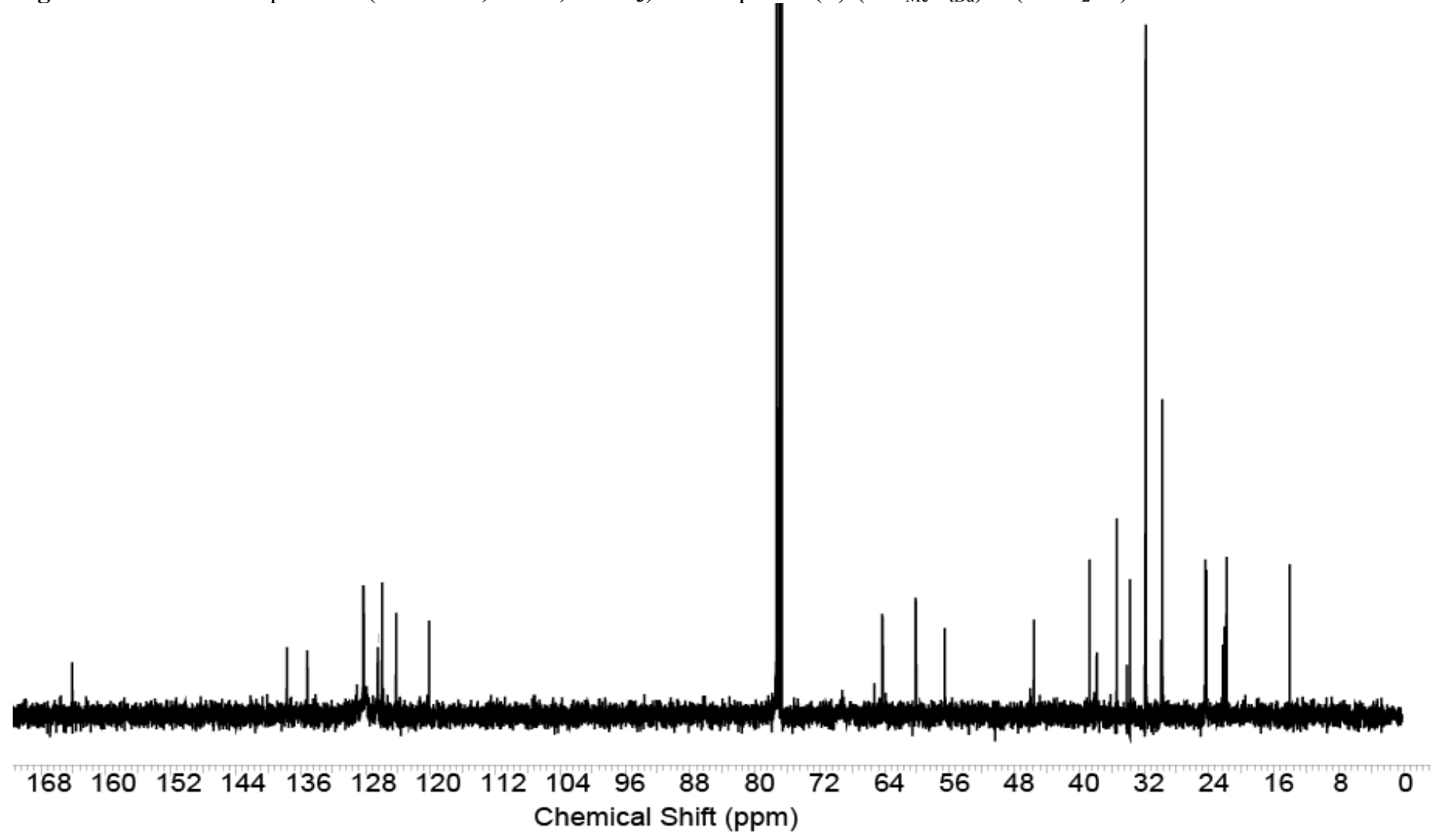

Figure S14. ${ }^{13} \mathrm{C}\left\{{ }^{1} \mathrm{H}\right\}$ NMR spectrum $\left(600 \mathrm{MHz}, 25{ }^{\circ} \mathrm{C}, \mathrm{CDCl}_{3}\right)$ of complex $7( \pm)-\left(\mathrm{NN}_{\mathrm{Me}} \mathrm{O}_{\mathrm{tBu}}\right) \mathrm{Zn}\left(\mathrm{OCH}_{2} \mathrm{Ph}\right)$. 


\section{B. Characterization of compounds 2-7 in the solid state}

Table S1. Selected crystallographic parameters.

\begin{tabular}{|c|c|c|c|c|c|c|}
\hline & 2 & 3 & 4 & 5 & 6 & 7 \\
\hline \multirow{3}{*}{$\begin{array}{l}\text { empirical } \\
\text { formula } \\
\text { fw }\end{array}$} & $\mathrm{C}_{25} \mathrm{H}_{42} \mathrm{~N}_{2} \mathrm{OZn}$ & $\mathrm{C}_{35} \mathrm{H}_{46} \mathrm{~N}_{2} \mathrm{OZn}$ & $\mathrm{C}_{60} \mathrm{H}_{92} \mathrm{~N}_{4} \mathrm{O}_{4} \mathrm{Zn}_{2}$ & $\mathrm{C}_{60} \mathrm{H}_{88} \mathrm{~N}_{4} \mathrm{O}_{4} \mathrm{Zn}_{2}$ & $\mathrm{C}_{80} \mathrm{H}_{96} \mathrm{~N}_{4} \mathrm{O}_{4} \mathrm{Zn}$ & $\mathrm{C}_{62} \mathrm{H}_{96} \mathrm{~N}_{4} \mathrm{O}_{4} \mathrm{Zn}$ \\
\hline & & & & & & 2 \\
\hline & 451.97 & 576.11 & 1064.11 & 1060.08 & 1308.34 & 1092.16 \\
\hline$T(\mathrm{~K})$ & 90 & 90 & 90 & 100 & 90 & 90 \\
\hline$a(\AA)$ & $10.8221(13)$ & $8.698(3)$ & $9.2510(16)$ & $10.1201(10)$ & $14.685(4)$ & $11.8172(5)$ \\
\hline$b(\AA)$ & $8.2063(10)$ & $13.248(4)$ & $17.559(3)$ & $12.8255(11)$ & $15.209(4)$ & $15.9905(9)$ \\
\hline$c(\AA)$ & $27.479(3)$ & $14.936(5)$ & $17.356(3)$ & $13.2607(13)$ & $18.275(5)$ & $16.8567(9)$ \\
\hline$\alpha(\mathrm{deg})$ & 90 & $67.192(6)$ & 90 & $117.778(4)$ & $109.488(6)$ & $89.9930(10)$ \\
\hline$\beta(\operatorname{deg})$ & $91.900(2)$ & $81.571(7)$ & $98.095(4)$ & $94.590(5)$ & $106.282(6)$ & $82.361(2)$ \\
\hline$\gamma(\operatorname{deg})$ & 90 & $74.512(6)$ & 90 & $104.696(4)$ & $103.098(6)$ & $72.8900(10)$ \\
\hline volume $\left(\AA^{3}\right)$ & $2439.1(5)$ & $1527.1(8)$ & $2791.2(8)$ & $1432.9(2)$ & $3454.6(16)$ & $3014.7(3)$ \\
\hline$Z$ & 4 & 2 & 2 & 1 & 2 & 2 \\
\hline cryst syst & monoclinic & triclinic & monoclinic & triclinic & triclinic & triclinic \\
\hline space group & $P 2{ }_{1} / \mathrm{c}$ & $P-1$ & $P 2{ }_{1} / \mathrm{c}$ & $P-1$ & $P-1$ & $P-1$ \\
\hline$d_{\text {calc }}\left(\mathrm{g} / \mathrm{cm}^{3}\right)$ & 1.231 & 1.253 & 1.266 & 1.228 & 1.258 & 1.203 \\
\hline$\mu(\mathrm{Mo} \mathrm{K} \alpha)\left(\mathrm{cm}^{-1}\right)$ & 10.24 & 8.33 & 9.08 & 8.84 & 7.47 & 8.43 \\
\hline $2 \theta_{\max }(\mathrm{deg})$ & 60.3 & 57.6 & 60.3 & 55.2 & 60.6 & 60.128 \\
\hline total no. of reflns & 65879 & 37176 & 41100 & 29647 & 91116 & 10665 \\
\hline $\begin{array}{l}\text { no. of indep } \\
\text { reflns }\left(R_{\text {int }}\right)\end{array}$ & $7202(0.0300)$ & $7914(0.0401)$ & $8209(0.0237)$ & $6620(0.036)$ & $20430(0.040)$ & 10637 \\
\hline $\begin{array}{l}\text { residuals (refined } \\
\text { on } F^{2} \text { ): } R_{1} ; w R_{2}\end{array}$ & $0.0320,0.0706$ & $0.0460,0.0846$ & $0.0282,0.0654$ & $0.0722,0.1398$ & $0.0557,0.0971$ & $0.0396,0.0826$ \\
\hline $\mathrm{GOF}$ & 1.112 & 1.022 & 1.033 & 1.06 & 1.02 & 0.916 \\
\hline $\begin{array}{l}\text { no. obsrvns }[I> \\
2 \sigma(I)]\end{array}$ & 9379 & 9412 & 9092 & 9932 & 9636 & 9561 \\
\hline $\begin{array}{l}\text { residuals (refined } \\
\text { on } F^{2}: R_{1}^{a} ; w R_{2}{ }^{b} \text { ) } \\
{ }^{a} R_{1}=\sum\left\|F_{\mathrm{o}}|-| F_{\mathrm{c}}\right\| \\
\left.w\left(F_{\mathrm{o}}\right)_{2}\right]^{1 / 2}\end{array}$ & $\begin{array}{l}0.0278,0.0688 \\
\left|F_{\mathrm{o}}\right|^{b} w R_{2}=[\Sigma\end{array}$ & $\begin{array}{c}0.0362,0.0801 \\
\left.v\left(F_{\mathrm{o}}^{2}-F_{\mathrm{c}}^{2}\right)^{2}\right) / \Sigma\end{array}$ & $0.0244,0.0629$ & $0.0542,0.1287$ & $0.0381,0.0886$ & $0.0612,0.0878$ \\
\hline
\end{tabular}




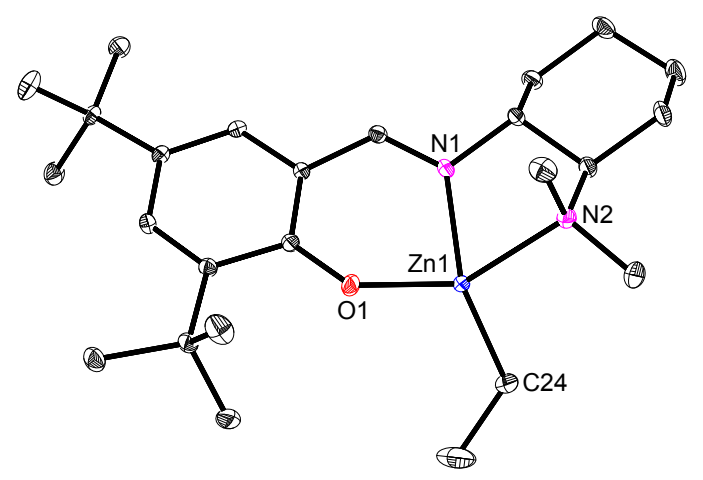

Figure S15. Molecular structure of complex 2 (depicted with thermal ellipsoids at 50\% probability and most $\mathrm{H}$ atoms omitted for clarity). Selected distances ( $\AA$ ) and angles (deg): N1-Zn1 2.0423(10), N2-Zn1 2.2916(10), O1Zn1 1.9750(9), C24-Zn1 1.9909(13), N1-Zn1-N2 76.87(4), N1-Zn1-O1 89.54(4), N1-Zn1-C24 139.76(5), N2-Zn1O1 121.52(4), N2-Zn1-C24 106.31(5), O1-Zn1-C24 118.88(5).

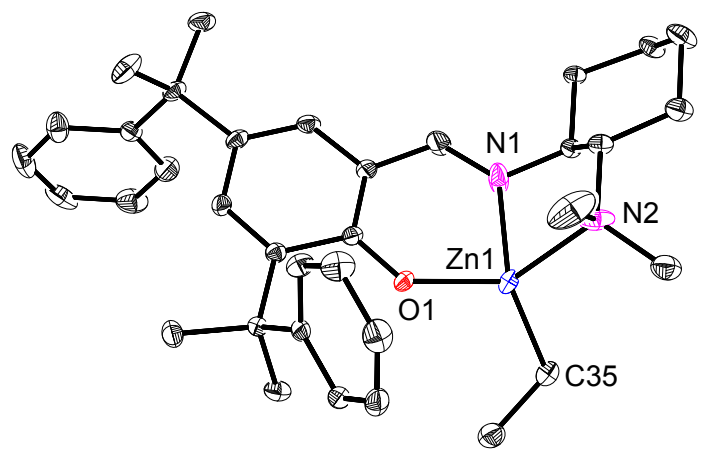

Figure S16. Molecular structure of complex 3 (depicted with thermal ellipsoids at 50\% probability and most $\mathrm{H}$ atoms omitted for clarity). Selected distances ( $\AA$ ) and angles (deg): N1-Zn1 2.0002(16), N2-Zn1 2.2854(16), O1Zn1 1.9674(12), C35-Zn1 1.9888(17), N1-Zn1-N2 77.84(6), N1-Zn1-O1 90.25(6), N1-Zn1-C35 135.06(7), N2-Zn1O1 118.89(6), N2-Zn1-C35 112.25(7), O1-Zn1-C35 117.41(6).

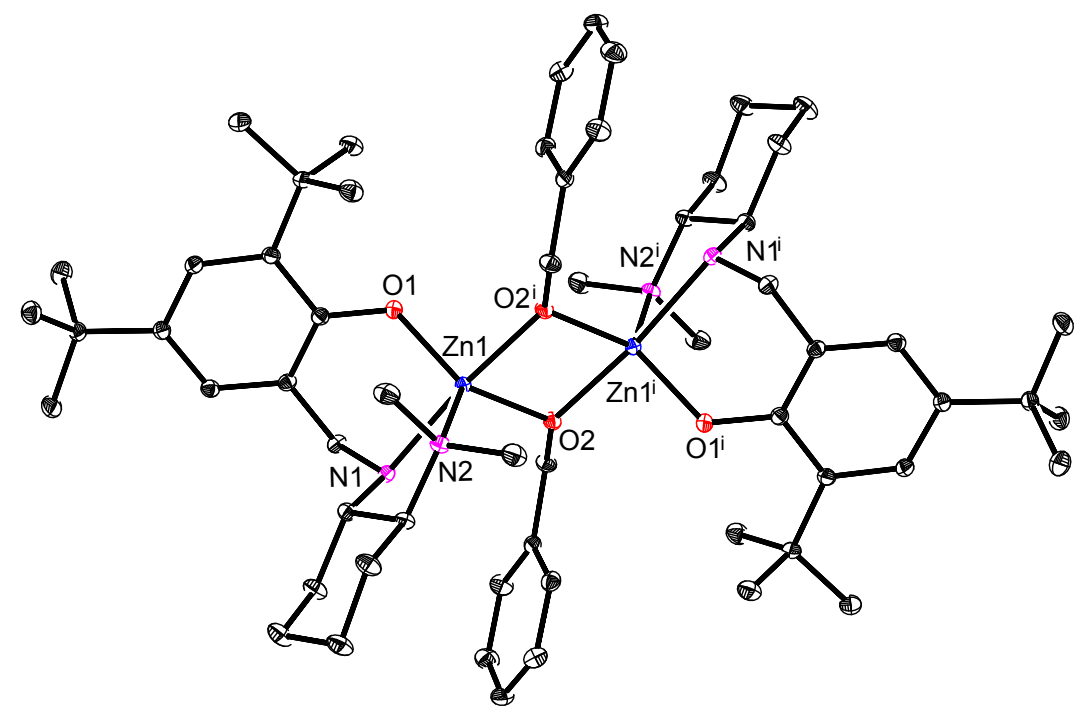

Figure S17. Molecular structure of complex 4 (depicted with thermal ellipsoids at 50\% probability and most $\mathrm{H}$ atoms omitted for clarity). Selected distances $(\AA)$ and angles (deg): N1-Zn1 2.2091(9), N2-Zn1 2.1845(9), O1-Zn1 1.9478(7), O2-Zn1 1.9852(8), O2 ${ }^{\mathrm{i}} \mathrm{Zn} 1$ 2.0852(7), N1-Zn1-N2 79.67(3), N1-Zn1-O1 89.86(3), N1-Zn1-O2 96.47(3), N1-Zn1-O2 ${ }^{\mathrm{i}}$ 173.45(3), N2-Zn1-O1 120.30(3), N2-Zn1-O2 ${ }^{\mathrm{i}}$ 96.29(3), N2-Zn1-O2 115.19(3), O1-Zn1-O2 124.38(3), O1-Zn1-O2 ${ }^{\mathrm{i}}$ 96.65(3), O2-Zn1-O2 ${ }^{\mathrm{i}}$ 80.50(3), Zn1-O2-Zn1 ${ }^{\mathrm{i}}$ 99.50(3). 


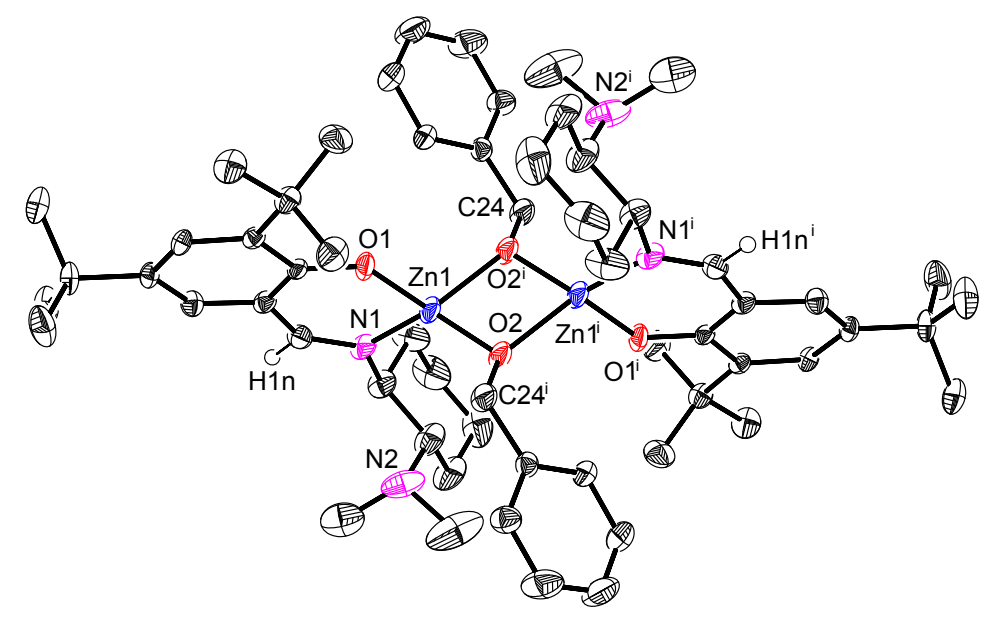

Figure S18. Molecular structure of complex 5 (depicted with thermal ellipsoids at $50 \%$ probability and most $\mathrm{H}$ atoms omitted for clarity). Selected distances ( $\AA$ ) and angles (deg): N1-Zn1 1.973(3), O1-Zn1 1.9213(19), O2-Zn1 1.962(2), O2 ${ }^{\mathrm{i}}-\mathrm{Zn} 1$ 1.9691(19), N1-Zn1-O1 96.68(9), N1-Zn1-O2 131.31(9), N1-Zn1-O2 ${ }^{\mathrm{i}}$ 126.76(9), O1-Zn1-O2 107.01(8), O1-Zn1-O2 ${ }^{\mathrm{i}}$ 112.48(8), O2-Zn1-O2 ${ }^{\mathrm{i}}$ 82.29(8), Zn1-O2-Zn1 ${ }^{\mathrm{i}}$ 97.71(8).

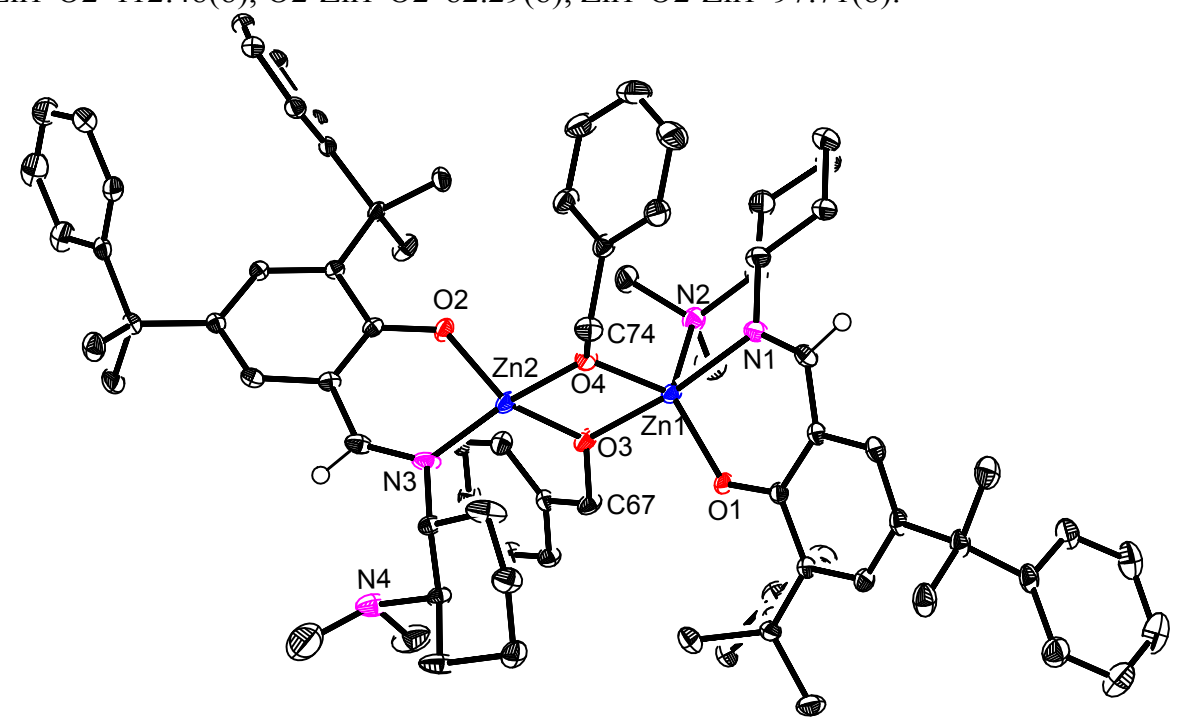

Figure S19. Molecular structure of complex 6 (depicted with thermal ellipsoids at 50\% probability and most $\mathrm{H}$ atoms omitted for clarity). Selected distances $(\AA)$ and angles (deg): N1-Zn1 2.0745(14), N2-Zn1 2.1932(15), O1Zn1 1.9539(12), O3-Zn1 2.0511(12), O4-Zn1 2.0097(12), N3-Zn2 1.9818(16), O2-Zn2 1.9418(12), O3-Zn2 1.9443(12), O4-Zn2 1.9469(12), N1-Zn1-N2 80.01(6), N1-Zn1-O1 89.71(5), N1-Zn1-O4 99.86(5), N1-Zn1-O3 171.13(5), O1-Zn1-O3 98.52(4), O1-Zn1-O4 119.57(5), N2-Zn1-O1 128.31(5), N2-Zn1-O4 112.10(5), N2-Zn1-O3 92.22(5), O3-Zn1-O4 79.00(5), Zn1-O3-Zn2 98.22(5), Zn1-O4-Zn2 99.55(5), N3-Zn2-O2 96.64(5), N3-Zn2-O3 126.29(6), N3-Zn2-O4 122.39(6), O2-Zn2-O3 116.95(5), O2-Zn2-O4 112.80(5), O3-Zn2-O4 83.19(5). 


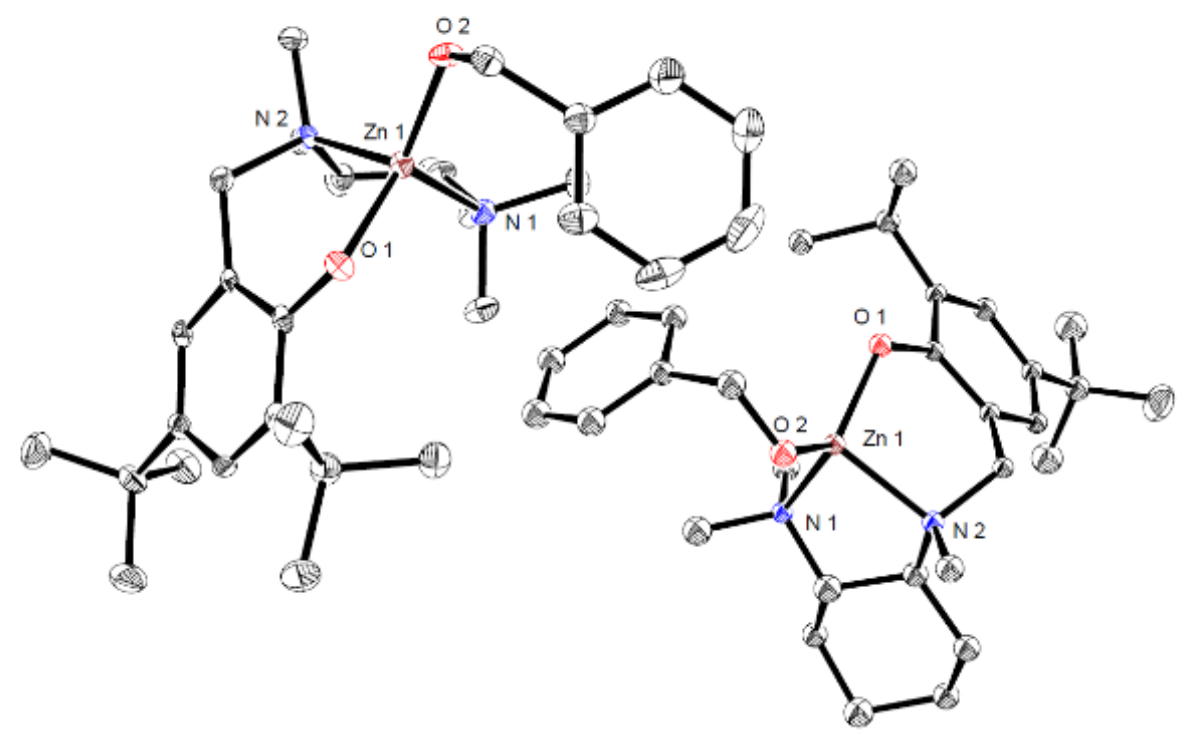

Figure S20. Molecular structure of complex rac-7 (depicted with thermal ellipsoids at 50\% probability and most $\mathrm{H}$ atoms as well as solvent molecules omitted for clarity). Selected distances ( $\AA$ ) and angles (deg): Zn1 O2 1.8757(17) Zn1 O1 1.9211(19), Zn1 N2 2.125(2), Zn1 N1 2.080(2). O2 Zn1 O1 124.44(8), O2 Zn1 N2 114.27(8), O2 Zn1 N1 111.34(9), O1 Zn1 N2 112.01(9), O1 Zn1 N1 100.75(8), N1 Zn1 N2 86.02(9), Zn1 O2 C23 116.84(4), O2 C23 C24 112.19(8). 


\section{Characterization of compounds 1-6 in solution by PGSE NMR}

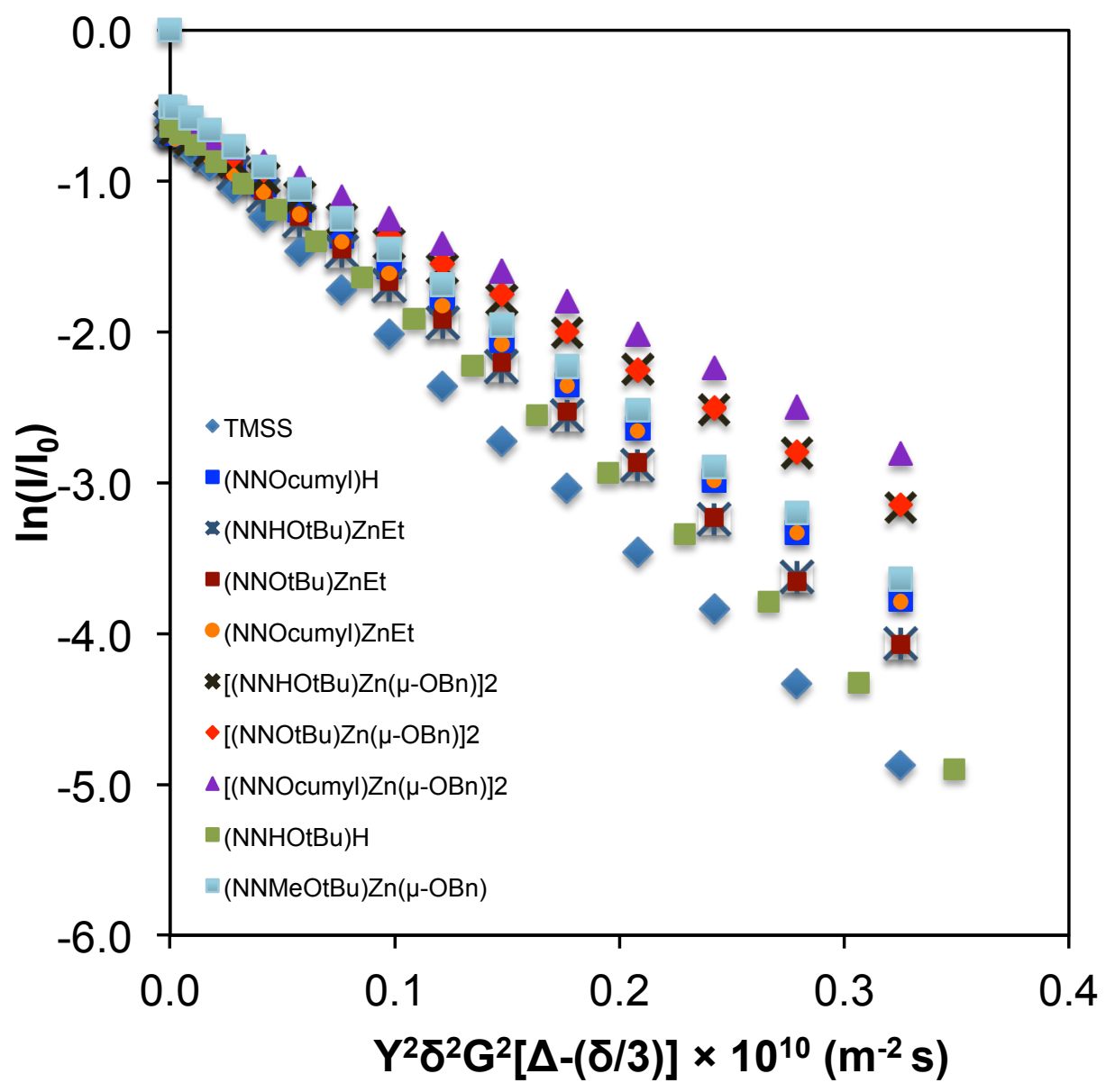

Figure S21. Plot of $\operatorname{In}\left(\mathrm{I} / \mathrm{I}_{0}\right)$ vs $\Upsilon^{2} \delta^{2} \mathrm{G}^{2}[\Delta-(\delta / 3)] \times 10^{10}\left(\mathrm{~m}^{-2} \mathrm{~s}\right)$ from PGSE experiments $\left(400 \mathrm{MHz}, \mathrm{CD}_{2} \mathrm{Cl}_{2}, 25{ }^{\circ} \mathrm{C}, \Delta\right.$ $=80 \mathrm{~ms}, \delta=1.1 \mathrm{~ms})$. The hydrodynamic radius $\left(\mathrm{r}_{\mathrm{H}}\right)$ of each compound was calculated by using the slopes $\left(D_{\mathrm{t}}\right)$ of the linear fits. $\mathrm{I}=$ intensity of the observed spin-echo, $\mathrm{I}_{0}=$ intensity of the spin-echo in the absence of gradients, $\mathrm{G}=$ varied gradient strength, $Y=$ gyromagnetic ratio $\left(2.675 \times 10^{8} \mathrm{rad} \mathrm{s}^{-1} \mathrm{~T}^{-1}\right), \delta=$ length of the gradient pulse, $\Delta=$ delay between the midpoints of the gradients.

Self-diffusion translational coefficients $\left(D_{\mathrm{t}}\right)$ were calculated graphically from the slopes of the linear best-fit lines. ${ }^{3}$ The $c^{s a} r_{H}{ }^{s a}$ value of each diffusing sample was estimated by the equation 1 which was derived from the advanced Stokes-Einstein equation 2. $r_{H}^{s a}$ was taken from the trend line of a plot, $c^{s a} r_{H}{ }^{s a}$ vs. $r_{H}^{s a}$, based on the equation 4 reported by Chen and coworkers. ${ }^{4}$

$$
c^{s a} r_{H}^{s a}=\frac{D_{t}^{s t} c^{s t} f_{s}^{s t} r_{H}^{s t}}{D_{t}^{s a} f_{s}^{s a}}
$$

$D_{\mathrm{t}}^{\text {st }}=$ translational diffusion coefficient of internal standard (TMSS, $D_{\mathrm{t}}^{\text {st }} \approx 14.2 \times 10^{-10} \mathrm{~m}^{2} \mathrm{~S}^{-1}, \mathrm{CD}_{2} \mathrm{Cl}_{2}, 25^{\circ} \mathrm{C}$ )

$c^{s t}=$ internal standard size correction factor (TMSS, $c^{s t}=5.1$ )

$f_{s}^{s t}=$ internal standard size and shape correction factor (TMSS, $f_{s}^{s t}=1$ )

$r_{H}^{s t}=$ internal standard hydrodynamic radius (TMSS, $4.51 \AA$ )

$D_{\mathrm{t}}^{\mathrm{sa}}=$ translational diffusion coefficient of sample $\left(\mathrm{CD}_{2} \mathrm{Cl}_{2}, 25^{\circ} \mathrm{C}\right)$

$c^{s a}=$ sample size correction factor

$f_{s}^{s a}=$ sample size and shape correction factor calculated from eq (3)

$r_{H}^{s a}=$ sample hydrodynamic radius 


$$
D_{t}=\frac{k T}{c f_{s} \pi \eta r_{H}}
$$

$k=$ Boltzmann constant $\left(k=1.38 \times 10^{-23} \mathrm{~m}^{2} \mathrm{~kg} \mathrm{~s}^{-1} \mathrm{~K}^{-1}\right)$

$T=$ absolute temperature $(\mathrm{K})$

$\eta=$ fluid viscosity $\left(\mathrm{CH}_{2} \mathrm{Cl}_{2}, \eta=0.0004 \mathrm{~kg} \mathrm{~s}^{-1} \mathrm{~m}^{-1}\right)$

$$
f_{s}=\frac{\sqrt{1-\left(\frac{b}{a}\right)^{2}}}{\left(\frac{b}{a}\right)^{\frac{2}{3}} \ln \frac{1+\sqrt{1-\left(\frac{b}{a}\right)^{2}}}{\left(\frac{b}{a}\right)}}
$$

$a=$ major semiaxes of a prolate ellipsoid estimated from X-ray crystal structure $b=$ minor semiaxes of a prolate ellipsoid estimated from X-ray crystal structure

$$
c r_{H}=\frac{6 r_{H}}{1+0.695\left(\frac{r_{\text {solv }}}{r_{H}}\right)^{2.234}}
$$

$r_{\text {solv }}=$ hydrodynamic radius of the solvent $\left(\mathrm{CH}_{2} \mathrm{Cl}_{2}=2.49 \AA\right)$ 
D. Variable temperature ${ }^{1} \mathrm{H}$ NMR spectra of complex 4

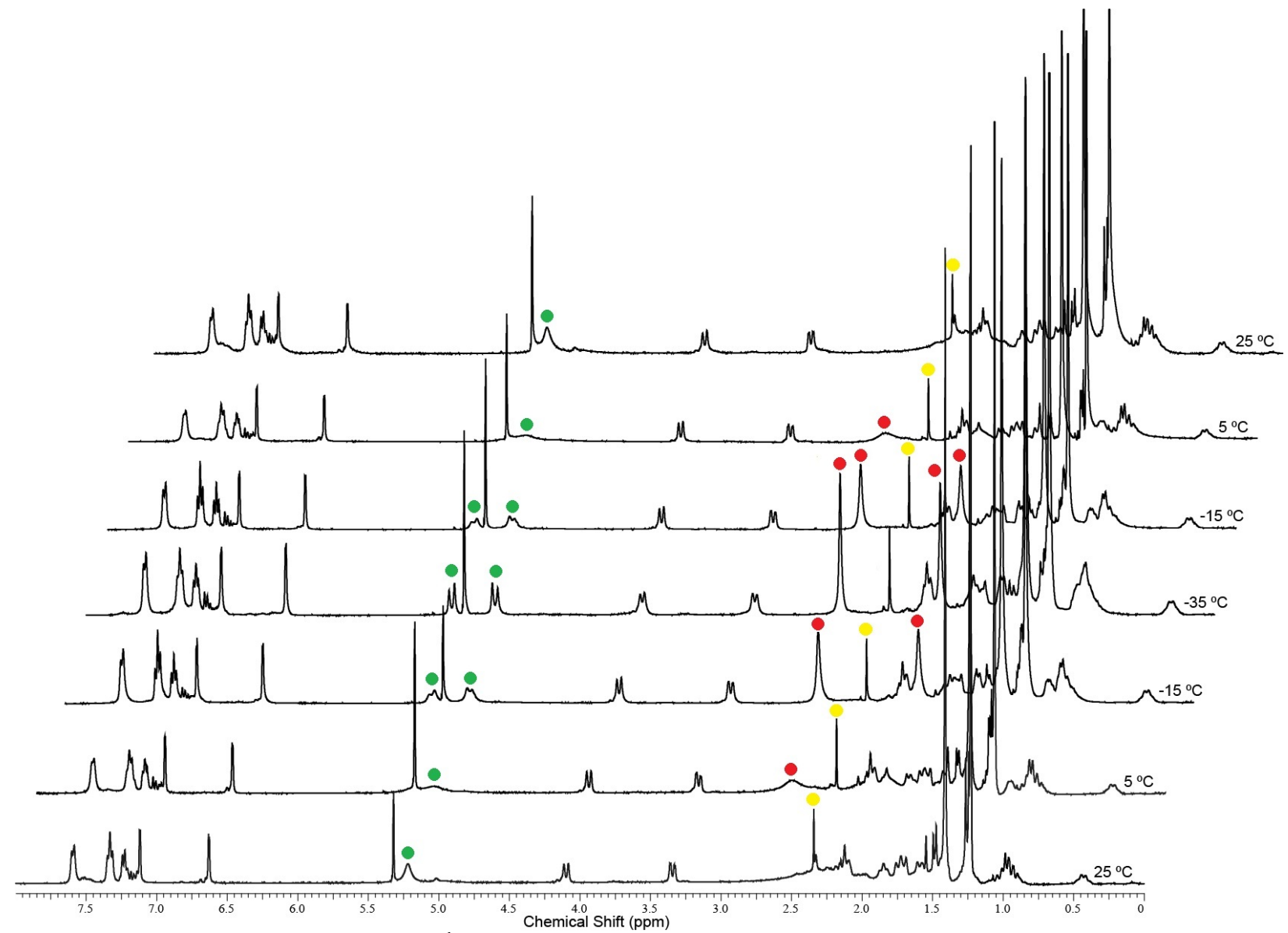

Figure S22. Variable temperature ${ }^{1} \mathrm{H}$ NMR spectra $\left(400 \mathrm{MHz}, \mathrm{CD}_{2} \mathrm{Cl}_{2}\right)$ of complex $4( \pm)-\left[\left(\mathrm{NN}_{\mathrm{H}} \mathrm{O}_{t-}\right.\right.$ $\left.\mathrm{Bu}) \mathrm{Zn}\left(\mathrm{OCH}_{2} \mathrm{Ph}\right)\right]_{2}$. ( - residual toluene, $\left.\bullet-\left(-\mathrm{CH}_{2}-\right), \bullet-\left(-\mathrm{CH}_{3}\right)_{2}\right)$. 


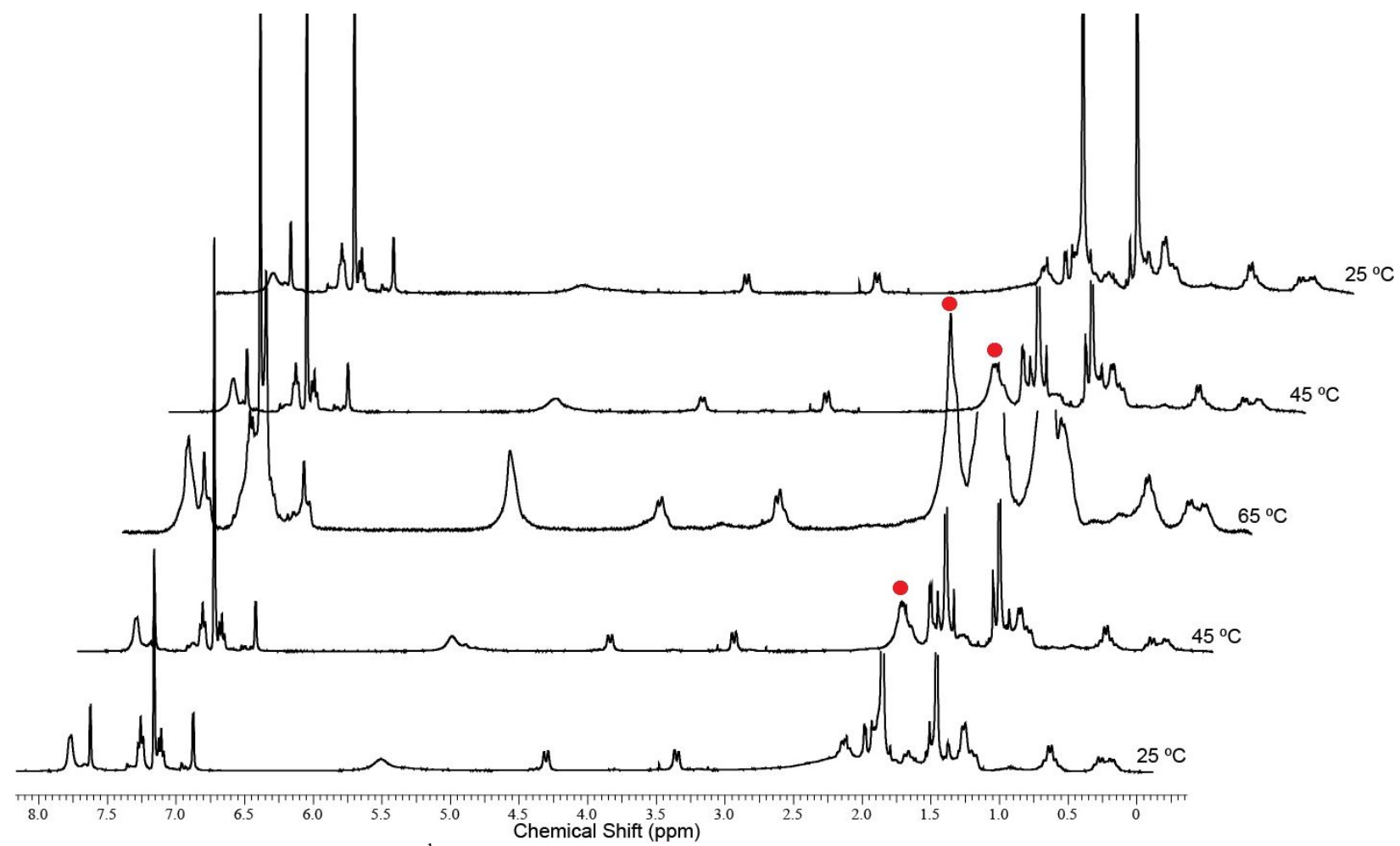

Figure S23. Variable temperature ${ }^{1} \mathrm{H}$ NMR spectra $\left(400 \mathrm{MHz}, \mathrm{C}_{6} \mathrm{D}_{6}\right)$ of complex $4( \pm)-\left[\left(\mathrm{NN}_{\mathrm{H}} \mathrm{O}_{t-\mathrm{Bu}}\right) \mathrm{Zn}\left(\mathrm{OCH}_{2} \mathrm{Ph}\right)\right]_{2}$. $\left(--\left(-\mathrm{CH}_{3}\right)_{2}\right)$. 
E. 2D NOESY spectra of complexes 4 and 5

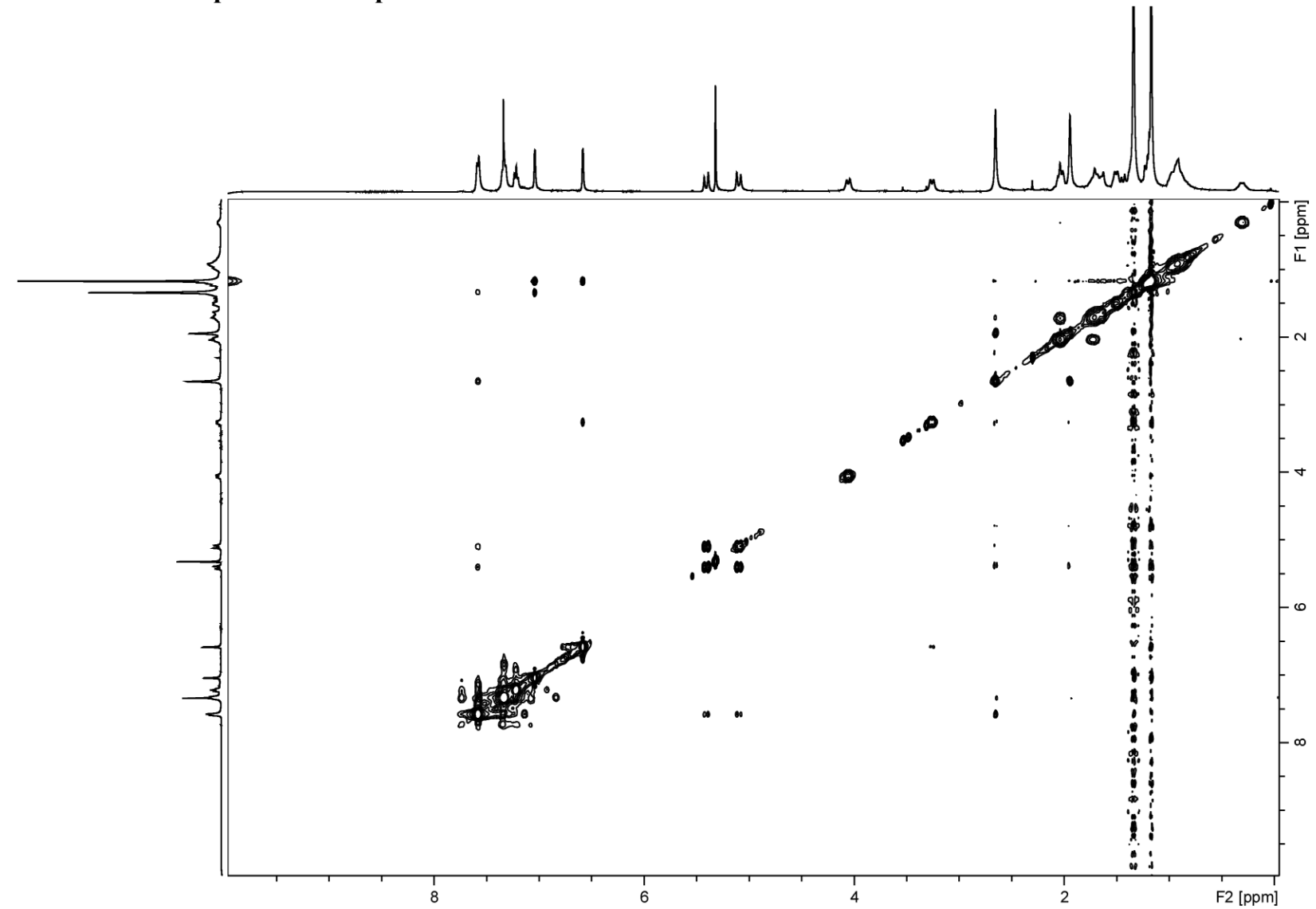

Figure S24. 2D NOESY full spectrum $\left(400 \mathrm{MHz}, \mathrm{CD}_{2} \mathrm{Cl}_{2},-35^{\circ} \mathrm{C}\right.$, mixing time $\left.=0.8 \mathrm{~s}\right)$ of complex $4( \pm)$ $\left[\left(\mathrm{NN}_{\mathrm{H}} \mathrm{O}_{t \mathrm{Bu}}\right) \mathrm{Zn}\left(\mathrm{OCH}_{2} \mathrm{Ph}\right)\right]_{2}$. 


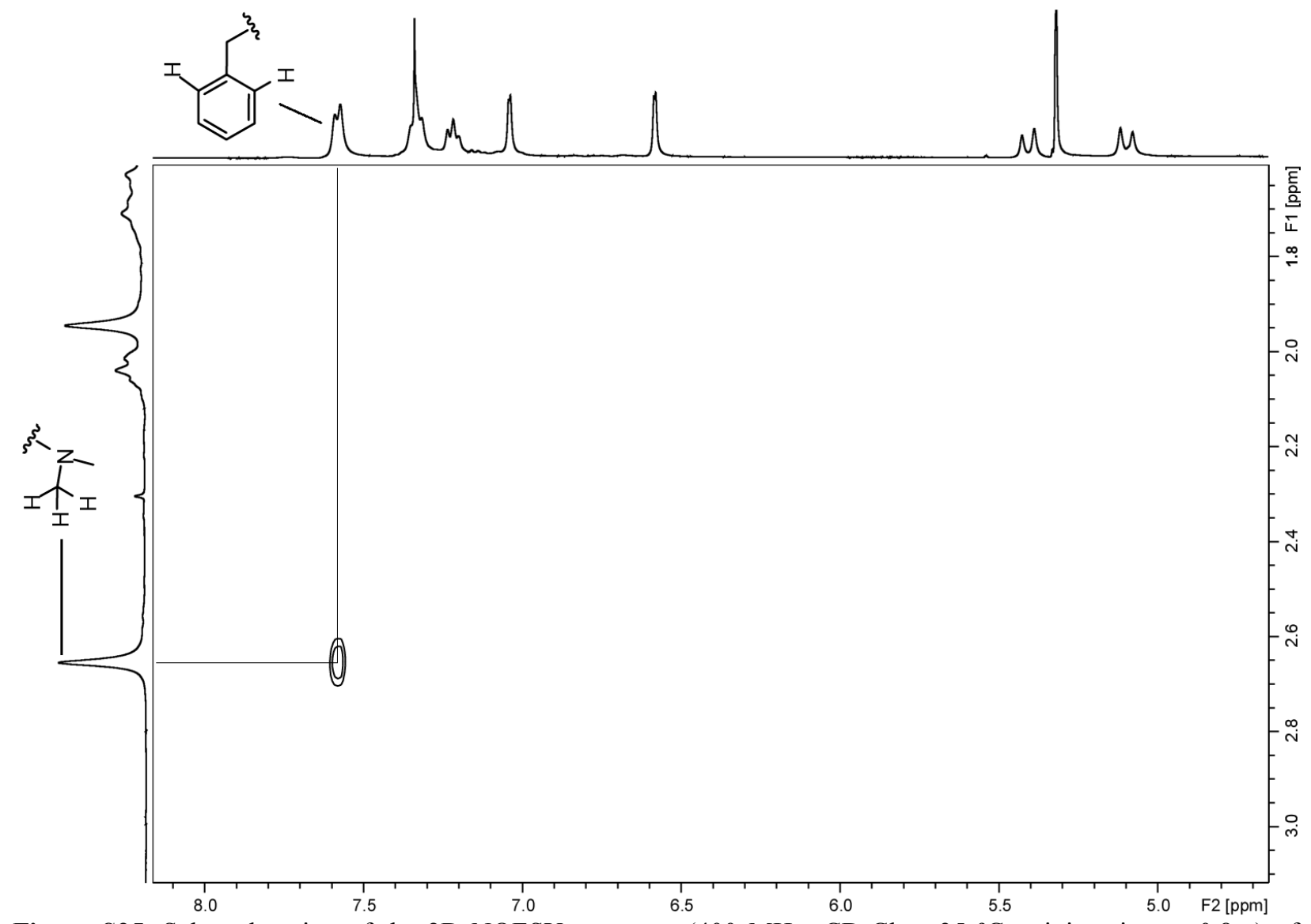

Figure S25. Selected region of the $2 \mathrm{D}$ NOESY spectrum $\left(400 \mathrm{MHz}, \mathrm{CD}_{2} \mathrm{Cl}_{2},-35{ }^{\circ} \mathrm{C}\right.$, mixing time $=0.8 \mathrm{~s}$ ) of complex $4( \pm)-\left[\left(\mathrm{NN}_{\mathrm{H}} \mathrm{O}_{t-\mathrm{Bu}}\right) \mathrm{Zn}\left(\mathrm{OCH}_{2} \mathrm{Ph}\right)\right]_{2}$. 


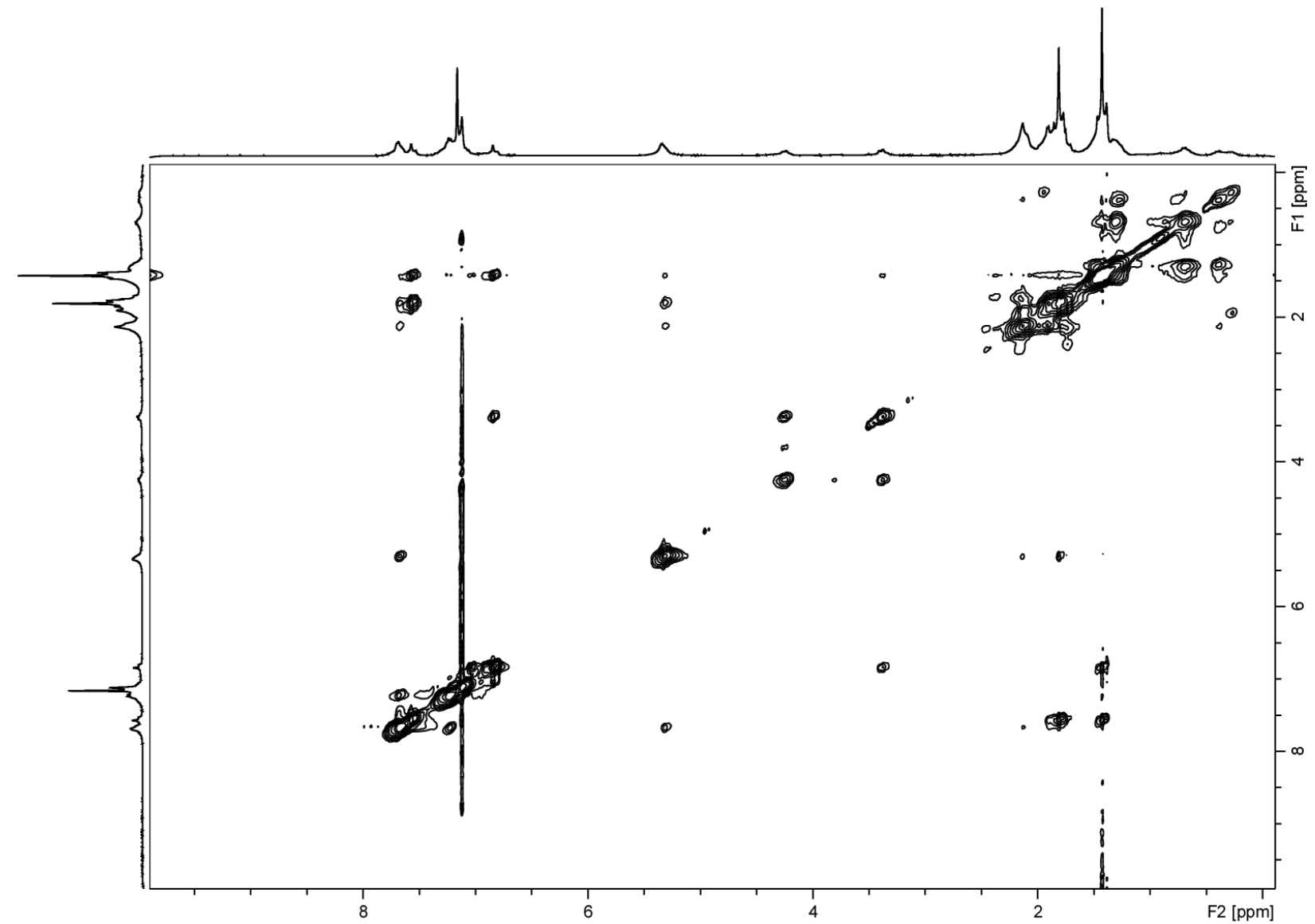

Figure S26. 2D NOESY full spectrum $\left(400 \mathrm{MHz}, \mathrm{C}_{6} \mathrm{D}_{6}, 65{ }^{\circ} \mathrm{C}\right.$, mixing time $\left.=1.0 \mathrm{~s}\right)$ of complex $4( \pm)$ $\left[\left(\mathrm{NN}_{\mathrm{H}} \mathrm{O}_{t \mathrm{Bu}}\right) \mathrm{Zn}\left(\mathrm{OCH}_{2} \mathrm{Ph}\right)\right]_{2}$. 


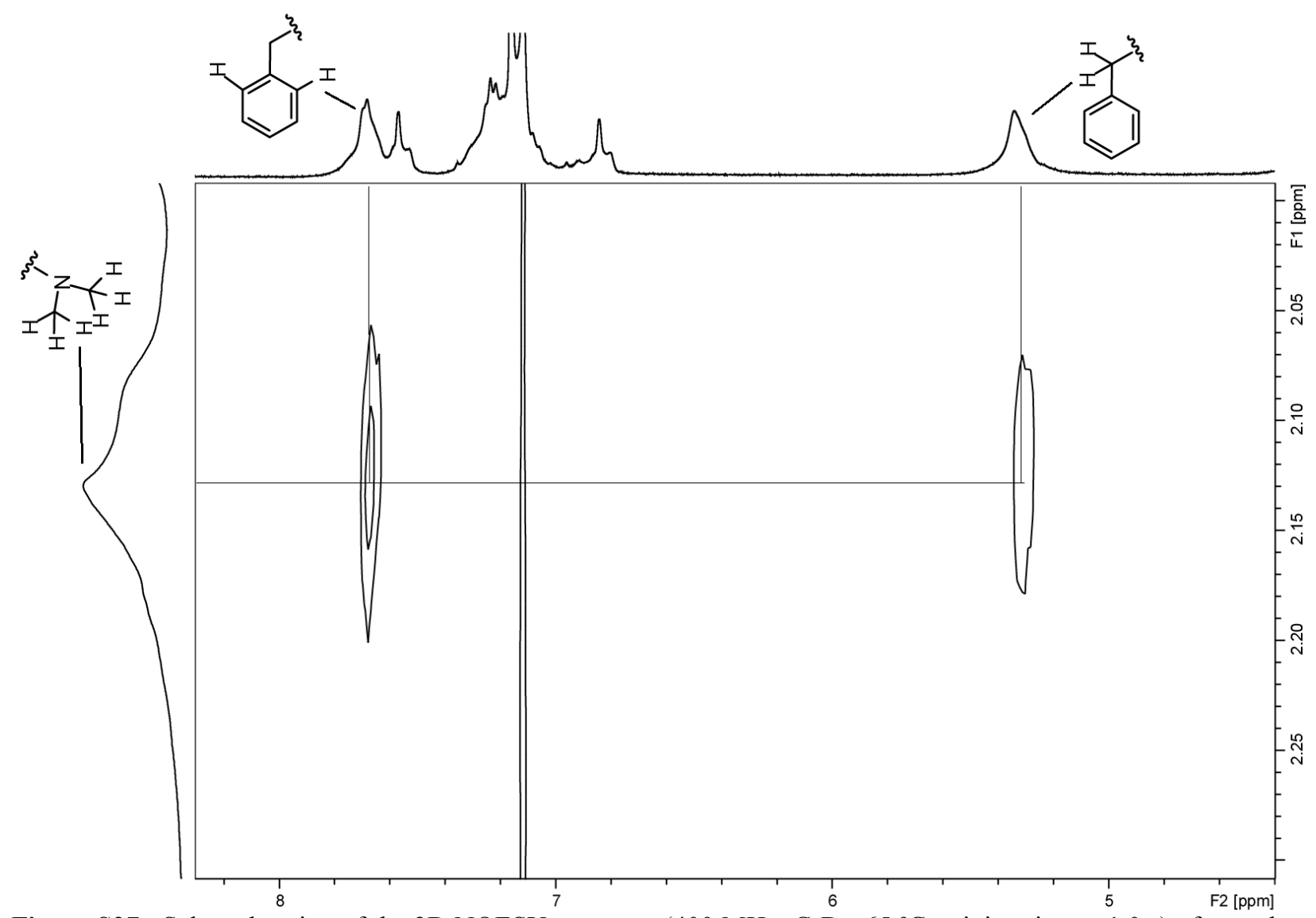

Figure S27. Selected region of the 2D NOESY spectrum $\left(400 \mathrm{MHz}, \mathrm{C}_{6} \mathrm{D}_{6}, 65^{\circ} \mathrm{C}\right.$, mixing time $\left.=1.0 \mathrm{~s}\right)$ of complex $4( \pm)-\left[\left(\mathrm{NN}_{\mathrm{H}} \mathrm{O}_{t-\mathrm{Bu}}\right) \mathrm{Zn}\left(\mathrm{OCH}_{2} \mathrm{Ph}\right)\right]_{2}$. 


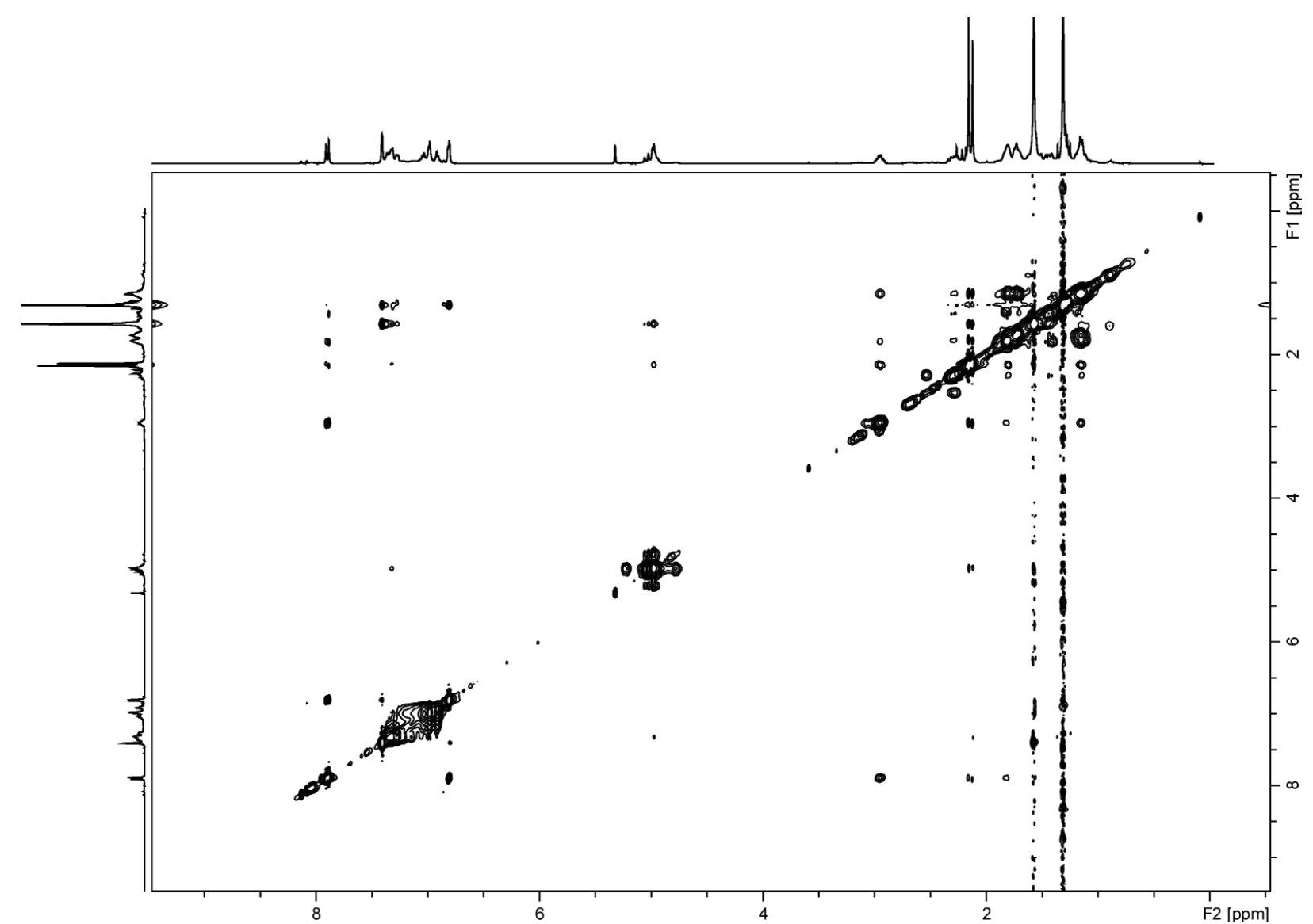

Figure S28. 2D NOESY full spectrum $\left(400 \mathrm{MHz}, \mathrm{CD}_{2} \mathrm{Cl}_{2}, 25{ }^{\circ} \mathrm{C}\right.$, mixing time $\left.=0.25 \mathrm{~s}\right)$ of complex $5( \pm)$ $\left[\left(\mathrm{NNO}_{t \mathrm{Bu}}\right) \mathrm{Zn}\left(\mathrm{OCH}_{2} \mathrm{Ph}\right)\right]_{2}$. 


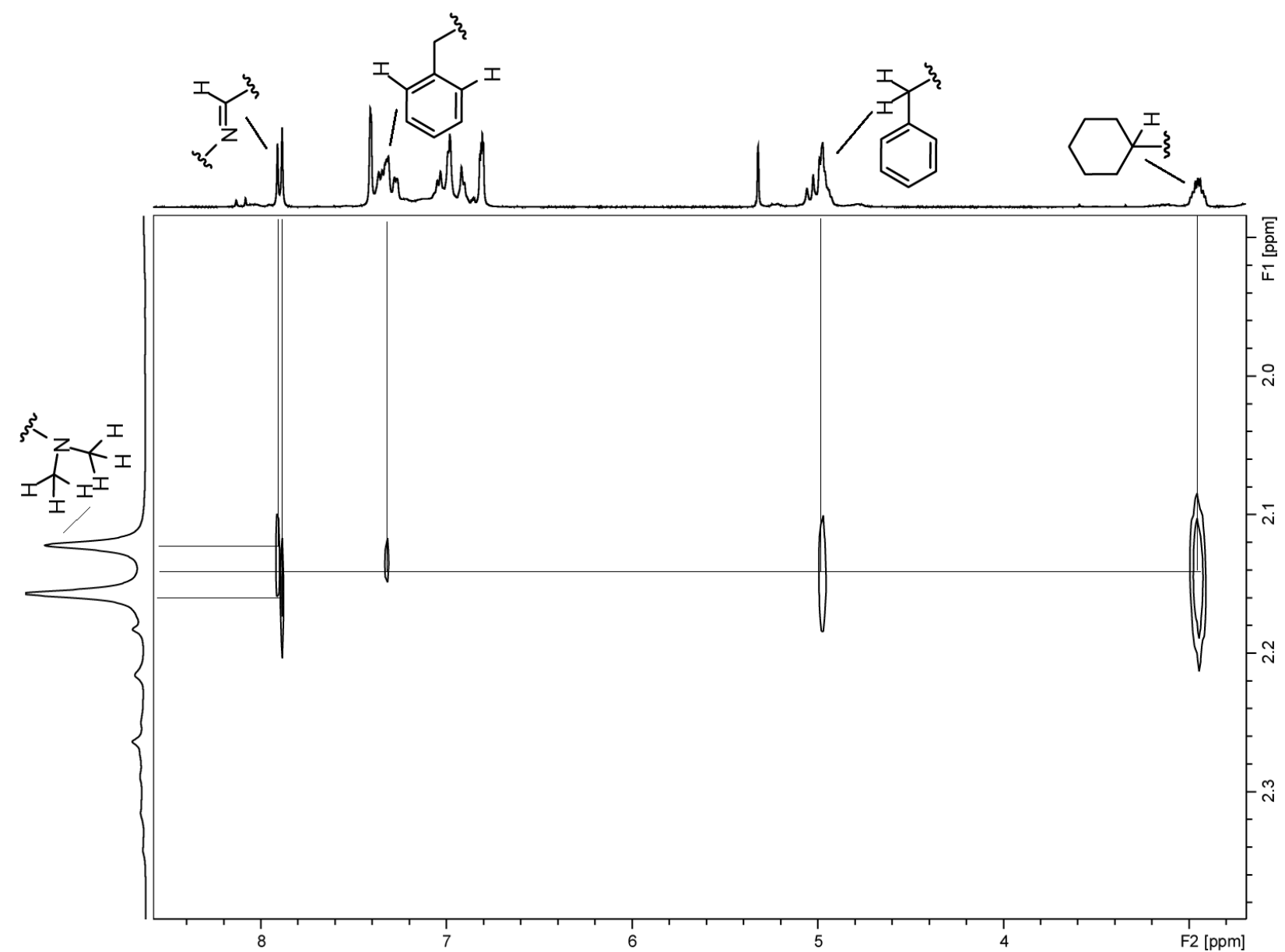

Figure S29. Selected region of the 2D NOESY spectrum $\left(400 \mathrm{MHz}, \mathrm{CD}_{2} \mathrm{Cl}_{2}, 25{ }^{\circ} \mathrm{C}\right.$, mixing time $\left.=0.25 \mathrm{~s}\right)$ of complex $5( \pm)-\left[\left(\mathrm{NNO}_{t-\mathrm{Bu}}\right) \mathrm{Zn}\left(\mathrm{OCH}_{2} \mathrm{Ph}\right)\right]_{2}$.

\section{F. Ring opening polymerization of rac-lactide catalyzed by complexes 1-3}

Table S2. Ring opening polymerization of $r a c$-lactide catalyzed by complexes 1-3.

\begin{tabular}{cccccccc}
\hline Entry & Cat & {$[\mathrm{M}]_{\mathrm{o}} /[\mathrm{I}]$} & Conv. $^{\mathrm{a}}(\%)$ & $\begin{array}{c}\mathrm{M}_{\mathrm{n}, \text { theo }} \mathrm{b}^{\mathrm{g}} \\
\mathrm{g} / \mathrm{mol})\end{array}$ & $\begin{array}{l}\mathrm{M}_{\mathrm{n}, \exp }{ }^{\mathrm{c}} \\
(\mathrm{g} / \mathrm{mol})\end{array}$ & $\begin{array}{l}\mathrm{M}_{\mathrm{w}, \exp }{ }^{\mathrm{c}} \\
(\mathrm{g} / \mathrm{mol})\end{array}$ & $\bigoplus_{\mathrm{M}}{ }^{\mathrm{c}}$ \\
\hline $1^{\mathrm{d}}$ & $\mathbf{1}$ & 200 & 72 & 20780 & $2.089 \times 10^{5}$ & $2.838 \times 10^{5}$ & 1.36 \\
$2^{\mathrm{d}}$ & $\mathbf{1}$ & 1000 & 82 & 118210 & $1.211 \times 10^{6}$ & $1.302 \times 10^{6}$ & 1.08 \\
3 & $\mathbf{2}$ & 200 & 46 & 13290 & $6.316 \times 10^{5}$ & $6.589 \times 10^{5}$ & 1.04 \\
4 & $\mathbf{2}$ & 1000 & 79 & 113890 & $1.523 \times 10^{6}$ & $1.622 \times 10^{6}$ & 1.06 \\
$5^{\mathrm{d}}$ & $\mathbf{3}$ & 200 & 38 & 10984 & $8.392 \times 10^{4}$ & $9.885 \times 10^{5}$ & 1.18 \\
$6^{\mathrm{d}}$ & $\mathbf{3}$ & 1000 & 78 & 112450 & $2.999 \times 10^{5}$ & $3.726 \times 10^{5}$ & 1.24 \\
\hline
\end{tabular}

All reactions were carried out in $\mathrm{CH}_{2} \mathrm{Cl}_{2}$ at $25^{\circ} \mathrm{C}$ in $20 \mathrm{~h}$ with $\mathrm{rac}$-lactide and $[\mathrm{Zn}]=0.7 \mathrm{mM}$. ${ }^{\mathrm{a}}$ Monomer conversion is determined by ${ }^{1} \mathrm{H}$ NMR spectroscopy. ${ }^{\mathrm{b}}$ Calculated from $[\mathrm{M}]_{\mathrm{o}} /[\mathrm{I}] \mathrm{x}$ monomer conversion $\times \mathrm{M}_{\mathrm{LA}}+\mathrm{M}_{\mathrm{C} 2 \mathrm{H} 6}\left(\mathrm{M}_{\mathrm{LA}}=\right.$ $144.13 \mathrm{~g} / \mathrm{mol}, \mathrm{M}_{\mathrm{C}^{2} \mathrm{H} 6}=30.07 \mathrm{~g} / \mathrm{mol}$ ). ${ }^{\circ}$ Determined by GPC-LALLS (gel permeation chromatography-low angle laser light scattering) $\left(\mathrm{dn} / \mathrm{dc}=0.044\right.$ for PLA). ${ }^{\mathrm{d}}$ Reactions were carried out in $\mathrm{CH}_{2} \mathrm{Cl}_{2}$ at $25^{\circ} \mathrm{C}$ in $44 \mathrm{~h}$ with $\mathrm{rac}$-lactide and $[\mathrm{Zn}]=0.7 \mathrm{mM}$. 


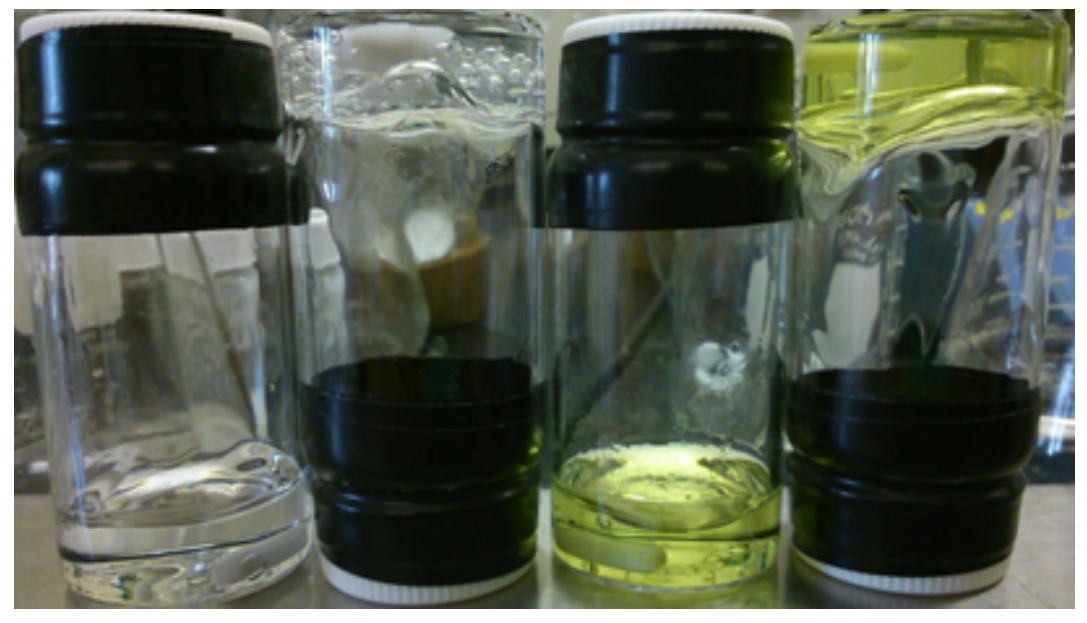

Figure S30. Gel formation during the polymerization reactions of rac-lactide catalyzed by complexes 1-3. 


\section{G. MALDI-TOF mass and ${ }^{1}$ H NMR spectra of PLA produced by complexes 4-6}

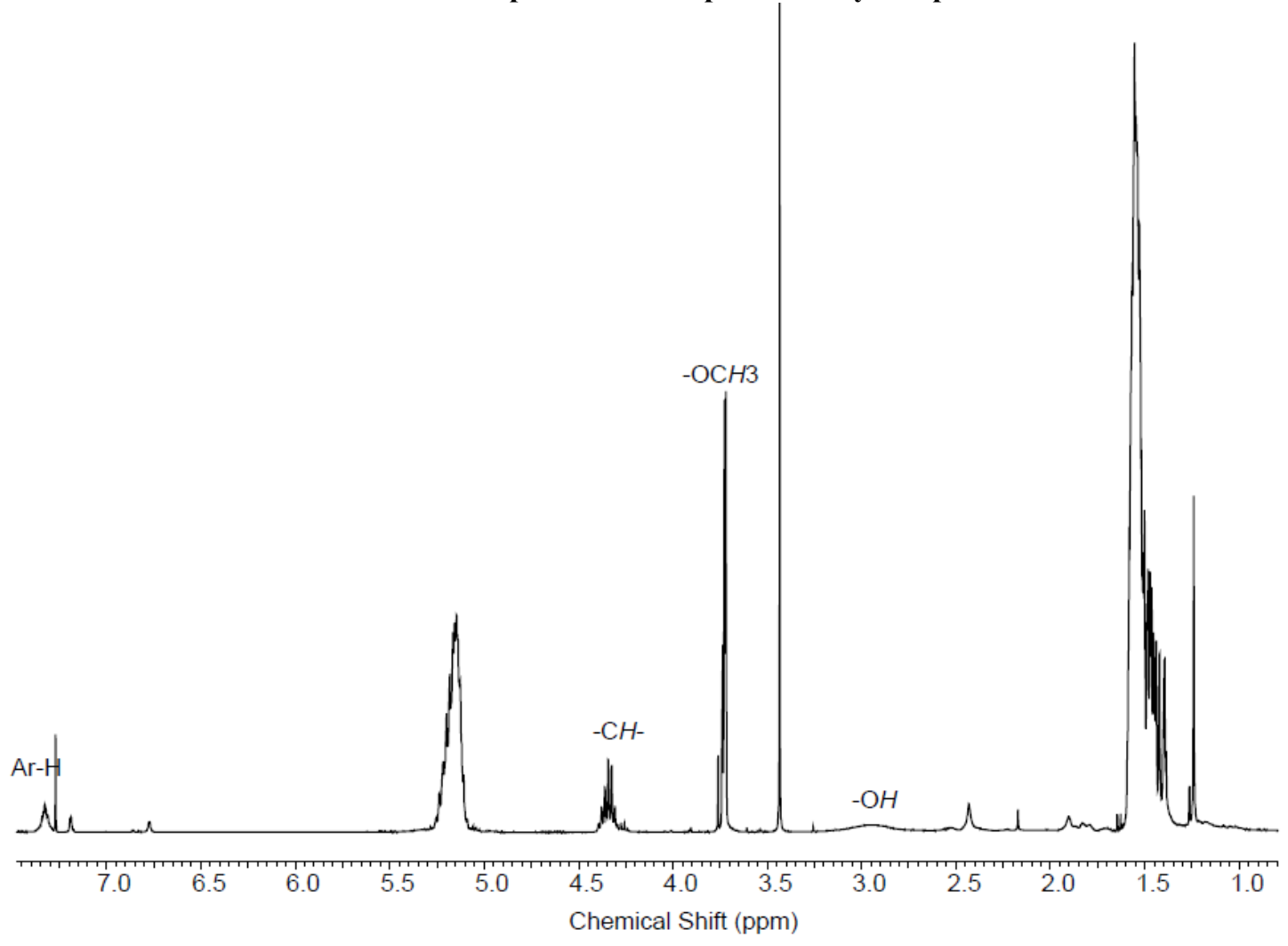

Figure S31. ${ }^{1} \mathrm{H}$ NMR spectrum of PLA generated by $4\left(400 \mathrm{MHz}, \mathrm{CDCl}_{3}, 25^{\circ} \mathrm{C}\right)$.

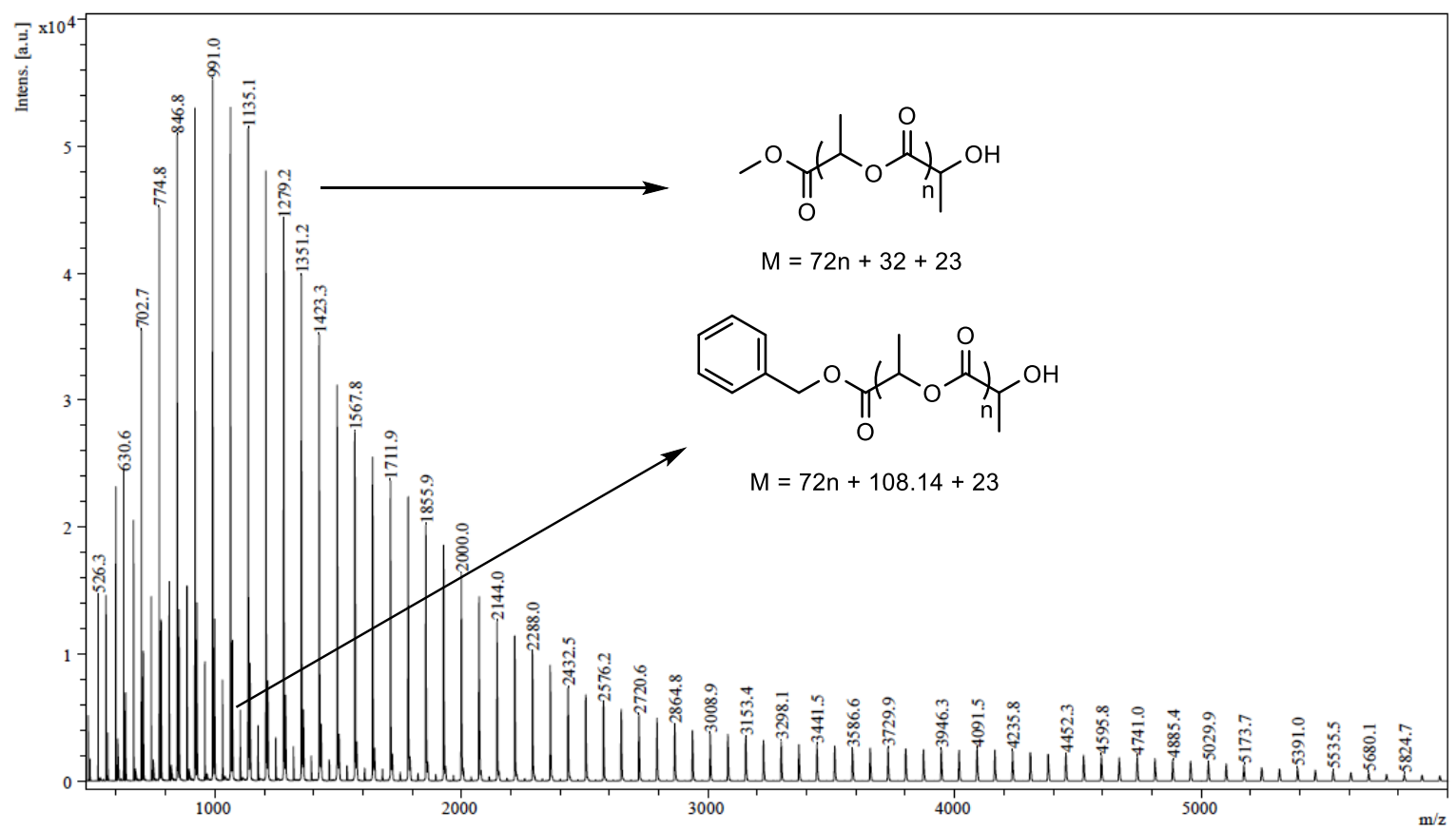

Figure S32. MALDI-TOF mass spectrum of PLA produced by complex 4 from ROP of $r a c$-lactide stopped after 5 minutes (2,5-dihydroxybenzoic acid with NaTFA). 


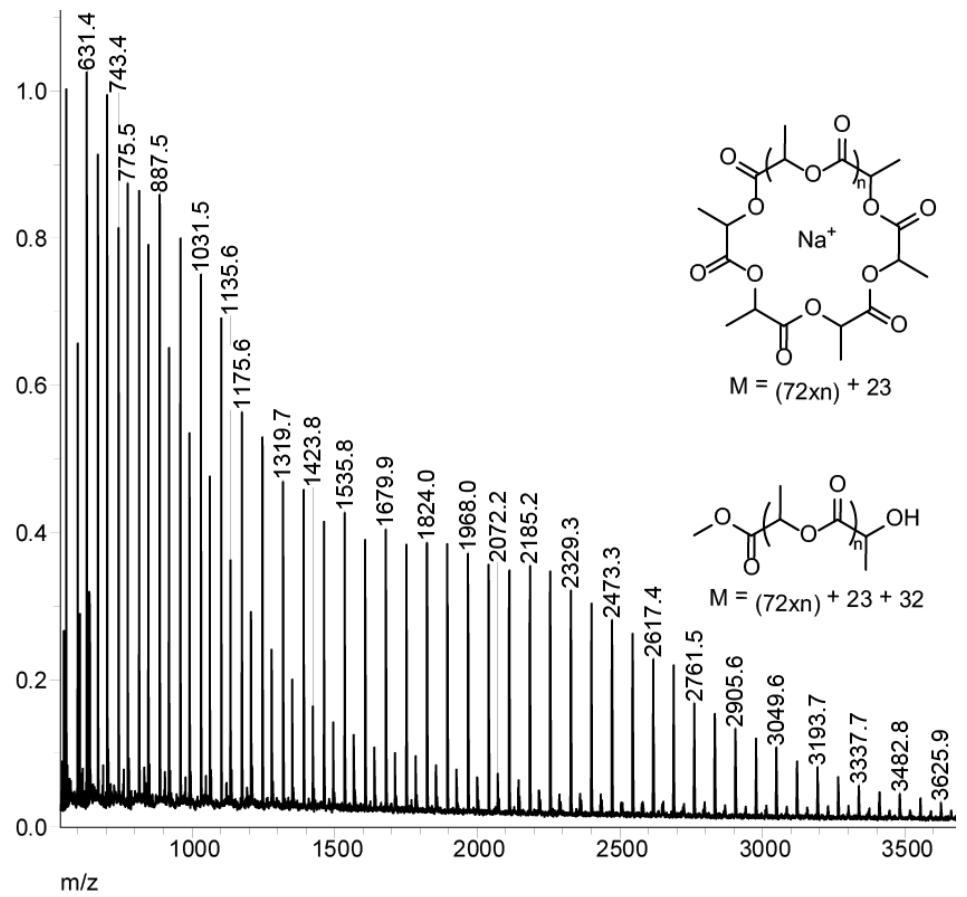

Figure S33. MALDI-TOF mass spectrum of PLA produced by complex 4 from ROP of rac-lactide stopped after 30 minutes (2,5-dihydroxybenzoic acid with NaTFA).

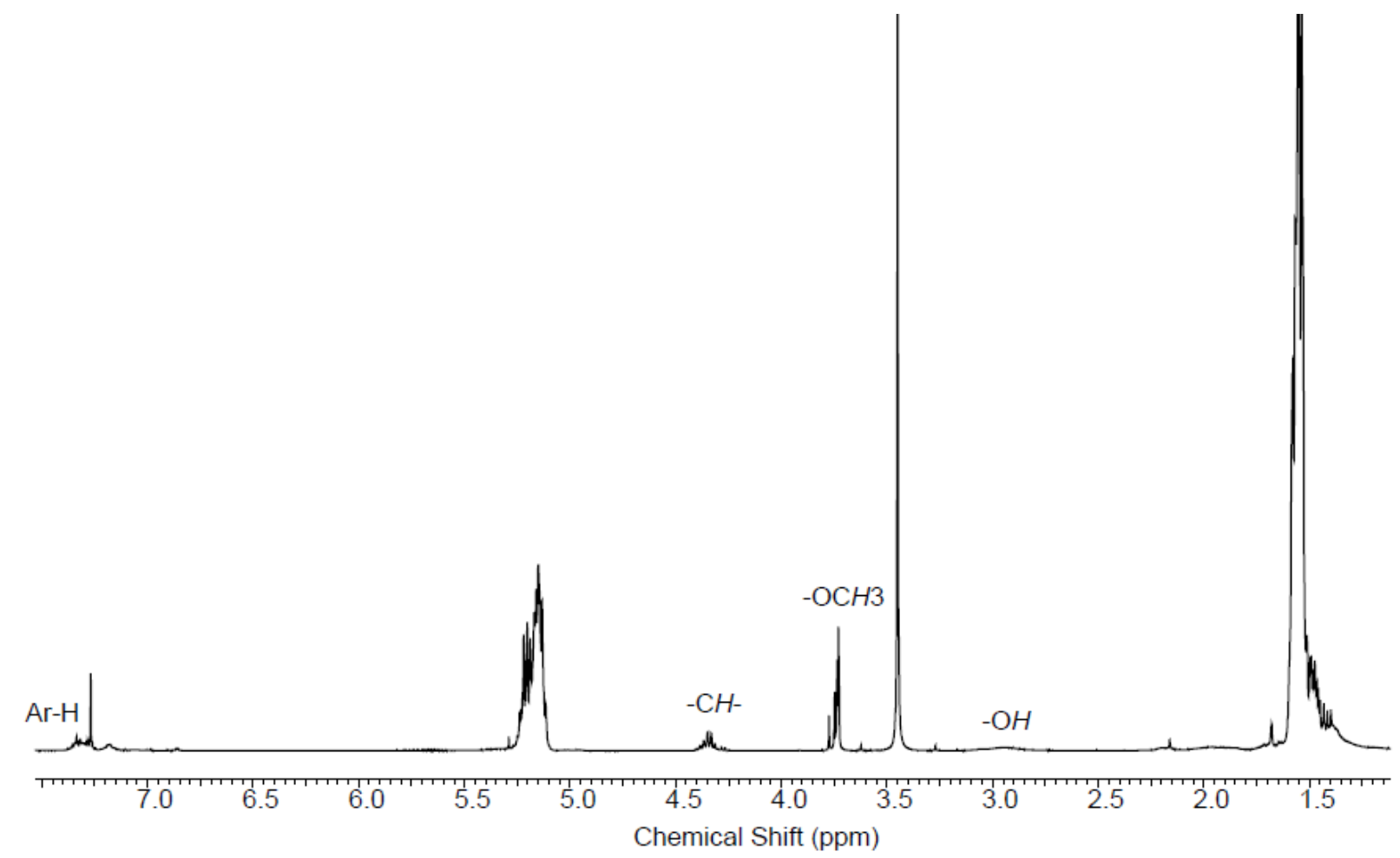

Figure S34. ${ }^{1} \mathrm{H}$ NMR spectrum of PLA generated by $5\left(400 \mathrm{MHz}, \mathrm{CDCl}_{3}, 25^{\circ} \mathrm{C}\right)$. 


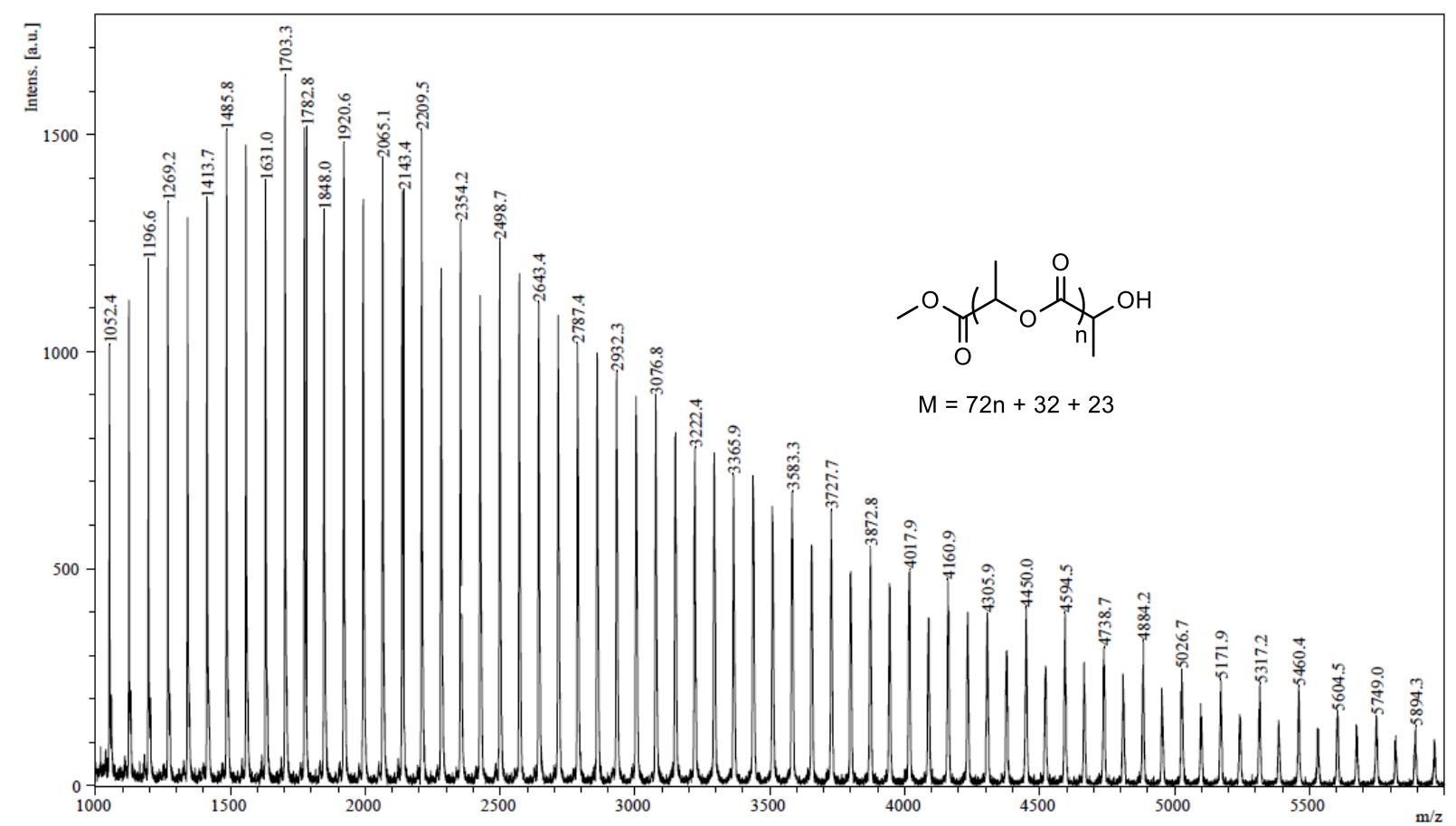

Figure S35. MALDI-TOF mass spectrum of PLA produced by complex 5 from ROP of rac-lactide $(2,5$ dihydroxybenzoic acid with NaTFA).

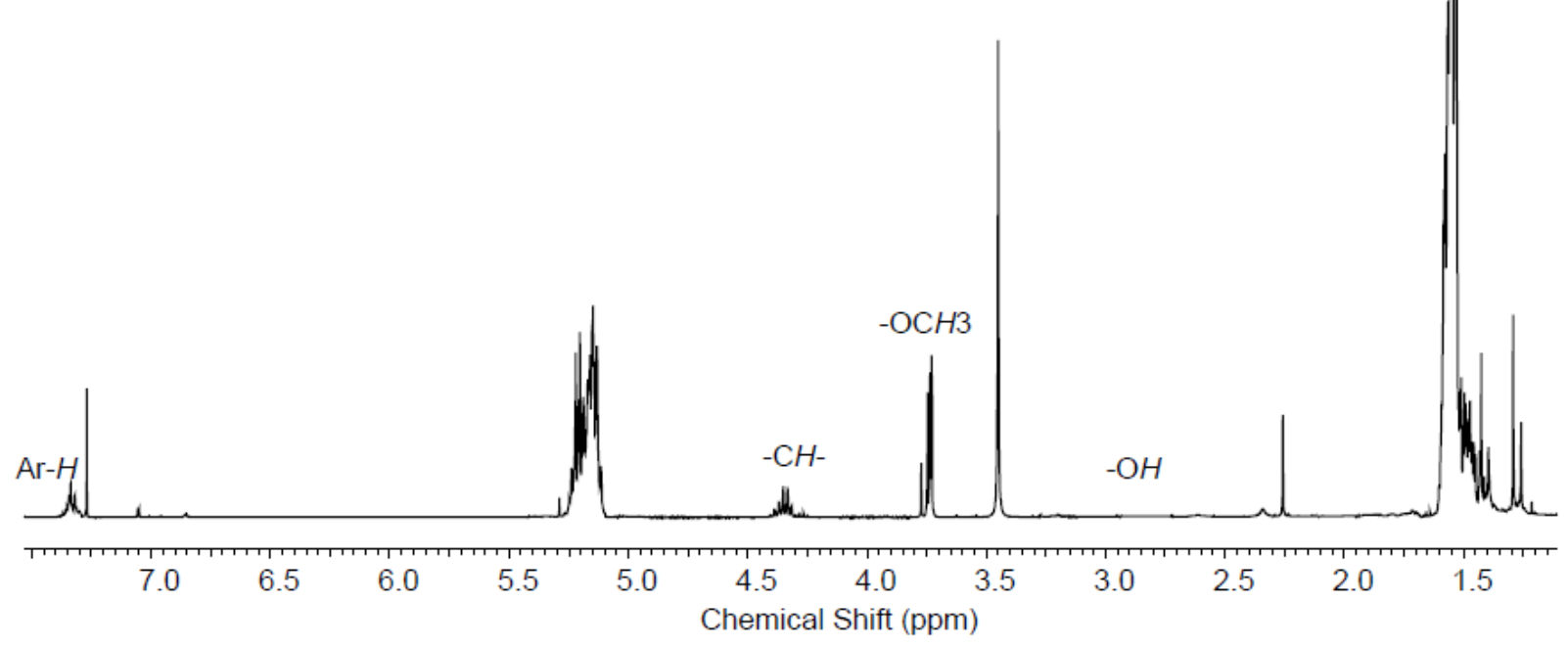

Figure S36. ${ }^{1} \mathrm{H}$ NMR spectrum of PLA generated by $6\left(400 \mathrm{MHz}, \mathrm{CDCl}_{3}, 25{ }^{\circ} \mathrm{C}\right)$. 


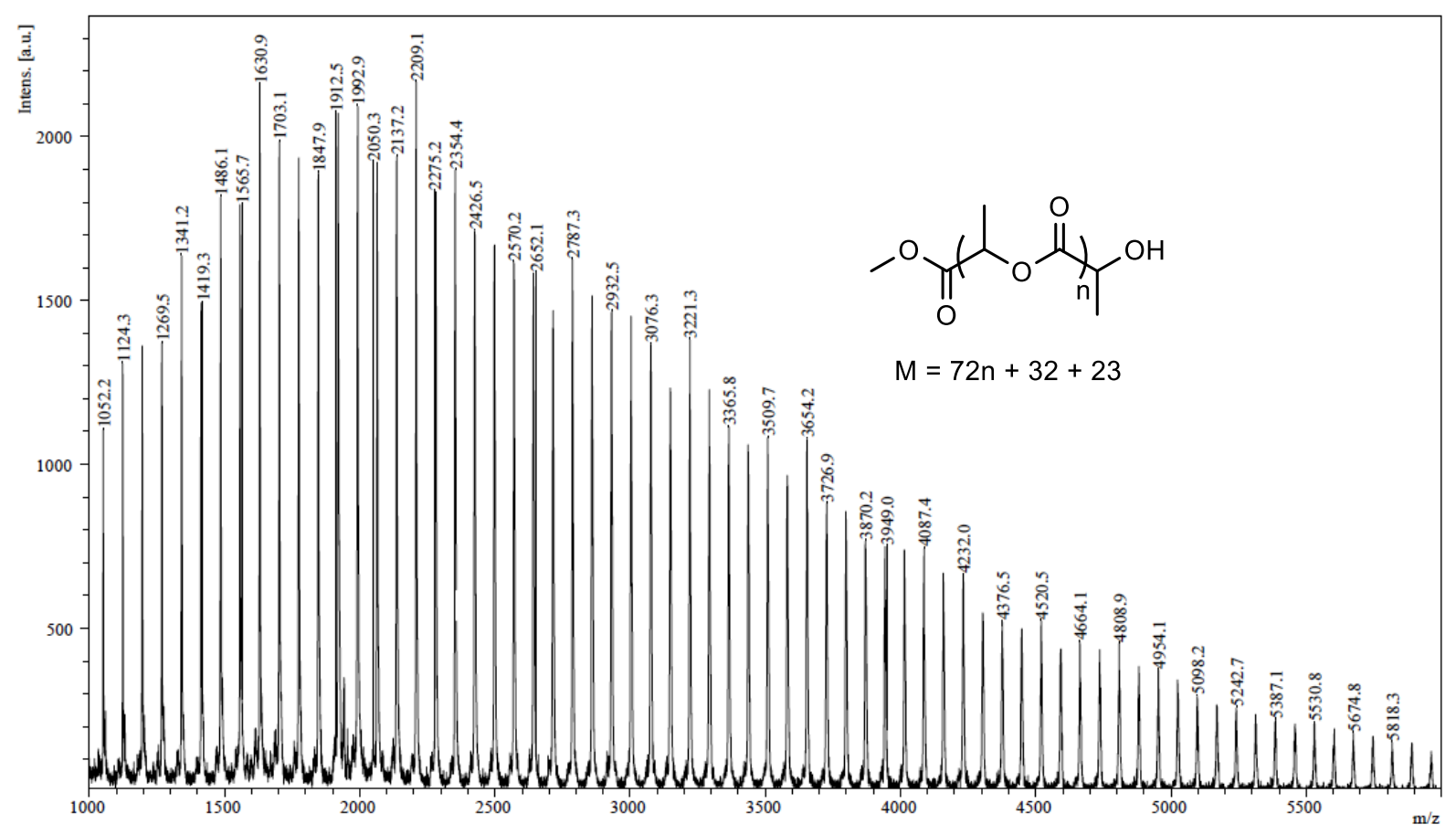

Figure S37. MALDI-TOF mass spectrum of PLA produced by complex 6 from ROP of rac-lactide (2,5dihydroxybenzoic acid with $\mathrm{NaCl}$ ).

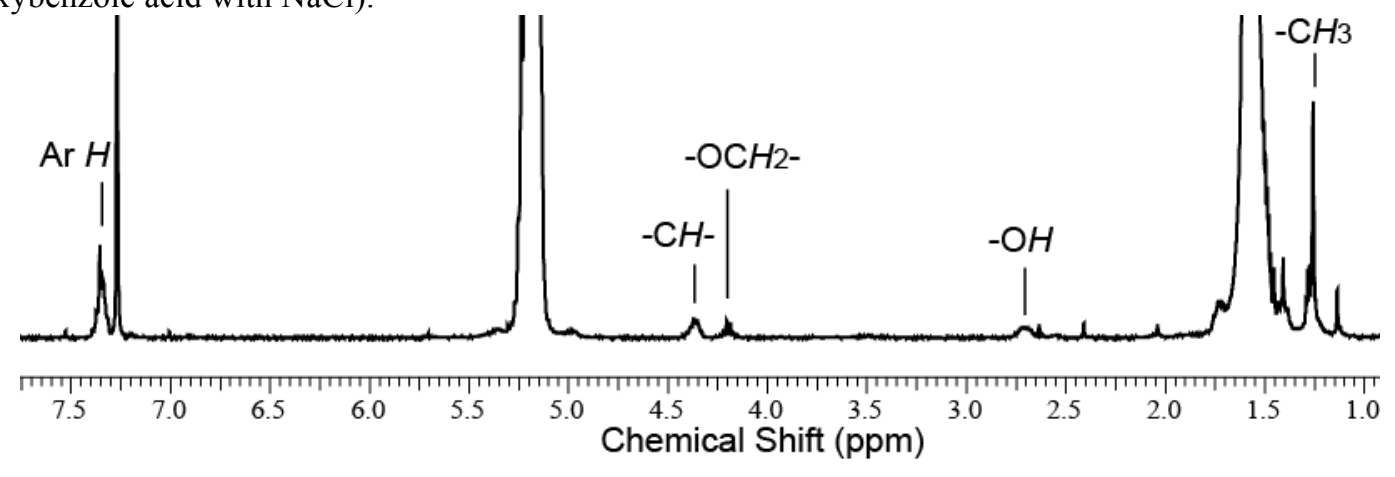

Figure S38. ${ }^{1} \mathrm{H}$ NMR spectrum of PLA generated by $\mathbf{4}$ and precipitated in ethanol $\left(400 \mathrm{MHz}, \mathrm{CDCl}_{3}, 25^{\circ} \mathrm{C}\right)$.

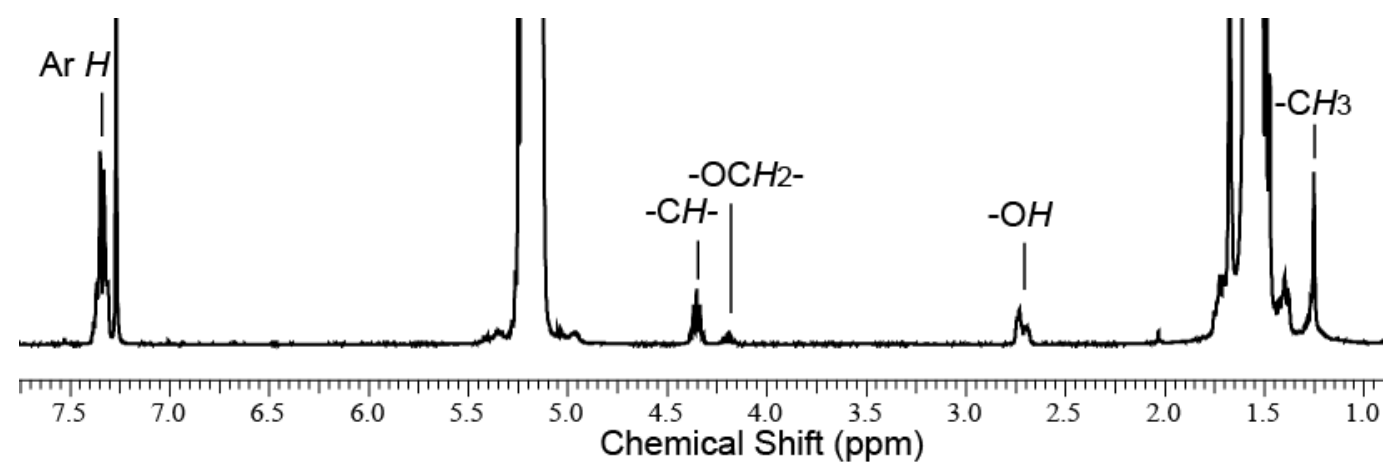


Figure S39. ${ }^{1} \mathrm{H}$ NMR spectrum of PLA generated by 5 and precipitated in ethanol $\left(400 \mathrm{MHz}, \mathrm{CDCl}_{3}, 25^{\circ} \mathrm{C}\right)$.

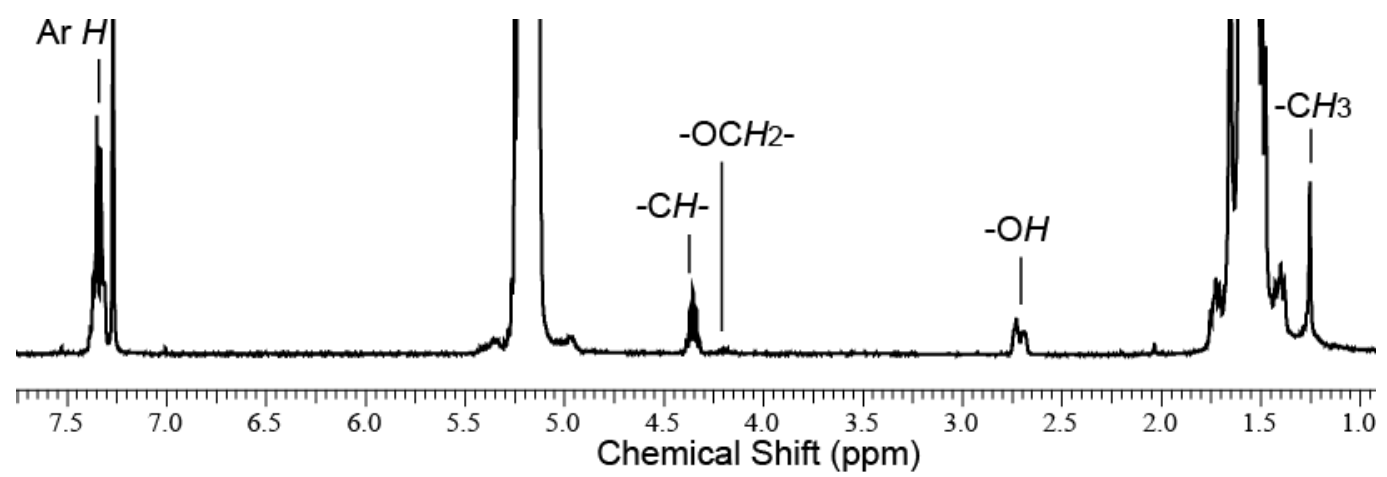

Figure S40. ${ }^{1} \mathrm{H}$ NMR spectrum of PLA generated by 6 and precipitated in ethanol $\left(400 \mathrm{MHz}, \mathrm{CDCl}_{3}, 25{ }^{\circ} \mathrm{C}\right)$. 


\section{H. Homonuclear decoupled ${ }^{1}$ H NMR spectra}

The $\mathrm{P}_{\mathrm{r}}$ and $\mathrm{P}_{\mathrm{m}}$ values were calculated from the following formulas which are based on tetrad probabilities in the polymerization of $r a c$-lactide as calculated from Bernoullian statistics. ${ }^{1}$ The assignment for each tetrad's chemical shift is based on the generally accepted values. ${ }^{2}$

Equations used:

$$
\begin{aligned}
& {[\mathrm{mmm}]=\left(\mathrm{P}_{\mathrm{m}}\right)^{2}+\mathrm{P}_{\mathrm{r}} \mathrm{P}_{\mathrm{m}} / 2} \\
& {[\mathrm{mmr}]=\mathrm{P}_{\mathrm{r}} \mathrm{P}_{\mathrm{m}} / 2} \\
& {[\mathrm{rmm}]^{*}=\mathrm{P}_{\mathrm{r}} \mathrm{P}_{\mathrm{m}} / 2} \\
& {[\mathrm{rmr}]^{*}=\left(\mathrm{P}_{\mathrm{r}}\right)^{2} / 2} \\
& {[\mathrm{mrm}]=\left(\left(\mathrm{P}_{\mathrm{r}}\right)^{2}+\mathrm{P}_{\mathrm{r}} \mathrm{P}_{\mathrm{m}}\right) / 2}
\end{aligned}
$$

*Effectively only these two equations are used in the calculations as the other peaks cannot be accurately integrated.

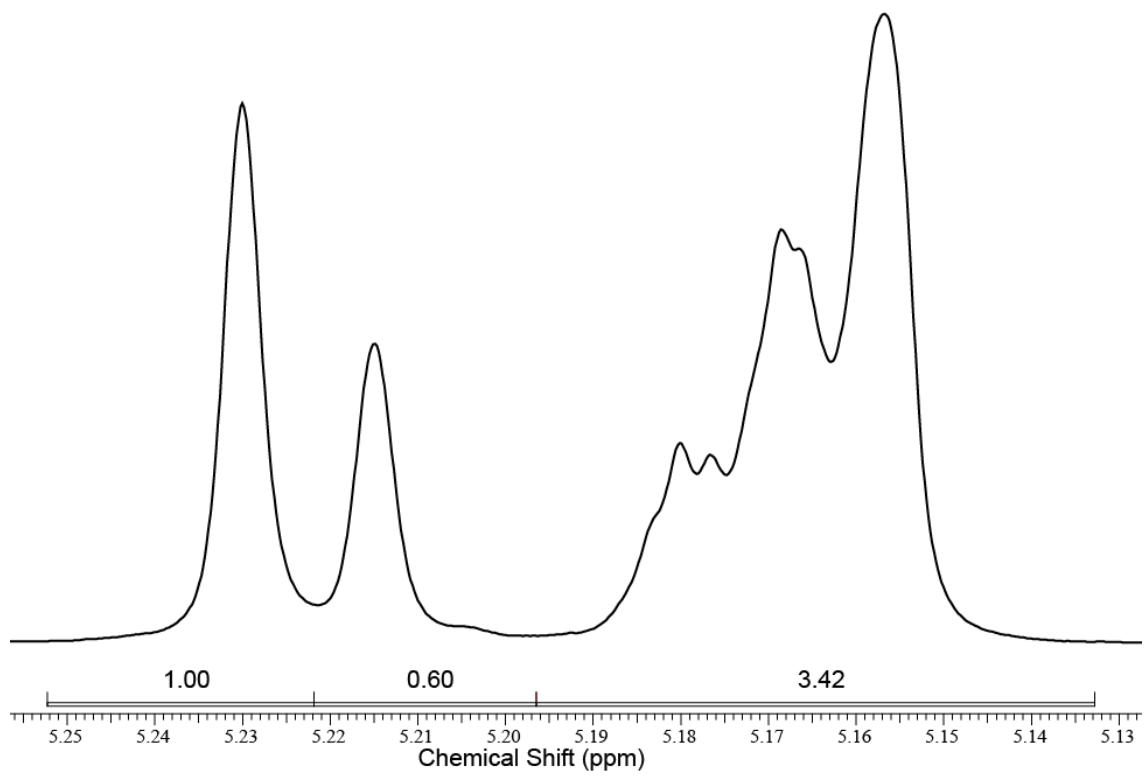

Figure S41. Homonuclear decoupled ${ }^{1} \mathrm{H}$ NMR spectrum of methine region of PLA prepared by complex $4\left(\mathrm{P}_{\mathrm{r}}=\right.$ $0.6)$. 


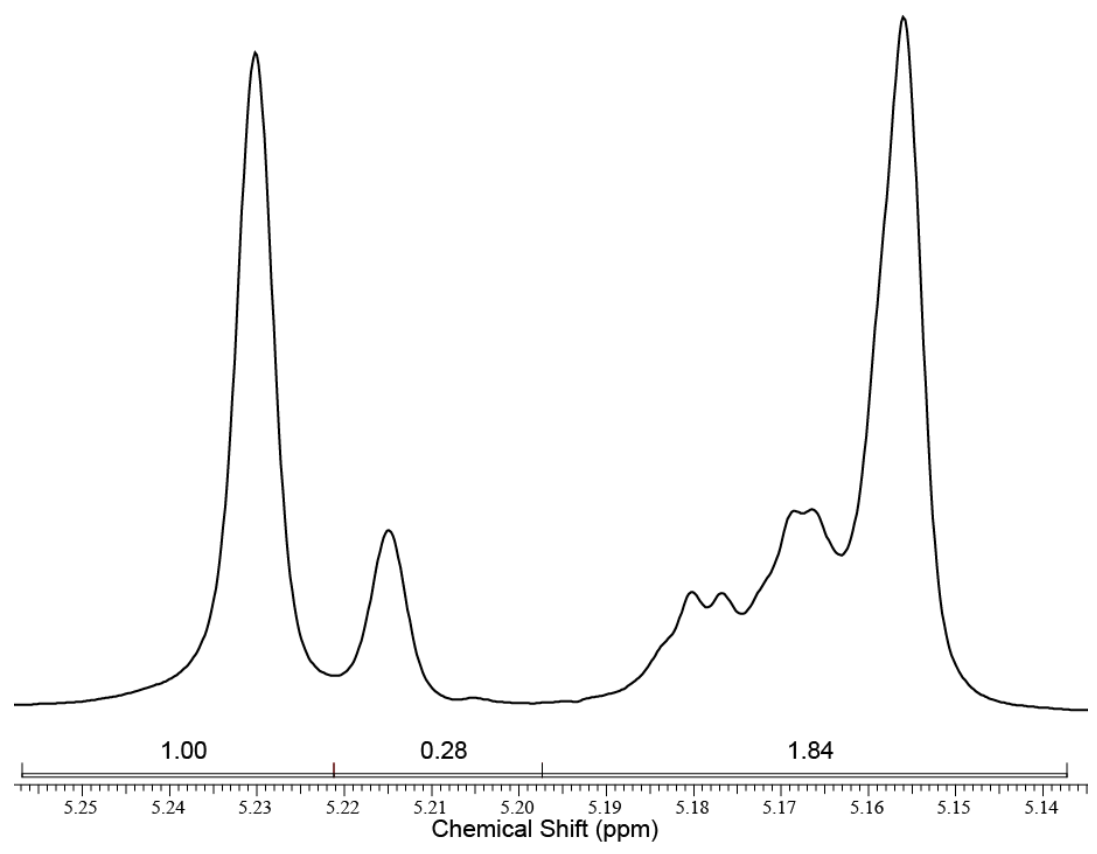

Figure S42. Homonuclear decoupled ${ }^{1} \mathrm{H}$ NMR spectrum of methine region of PLA prepared by complex $5\left(\mathrm{P}_{\mathrm{r}}=\right.$ $0.80)$.

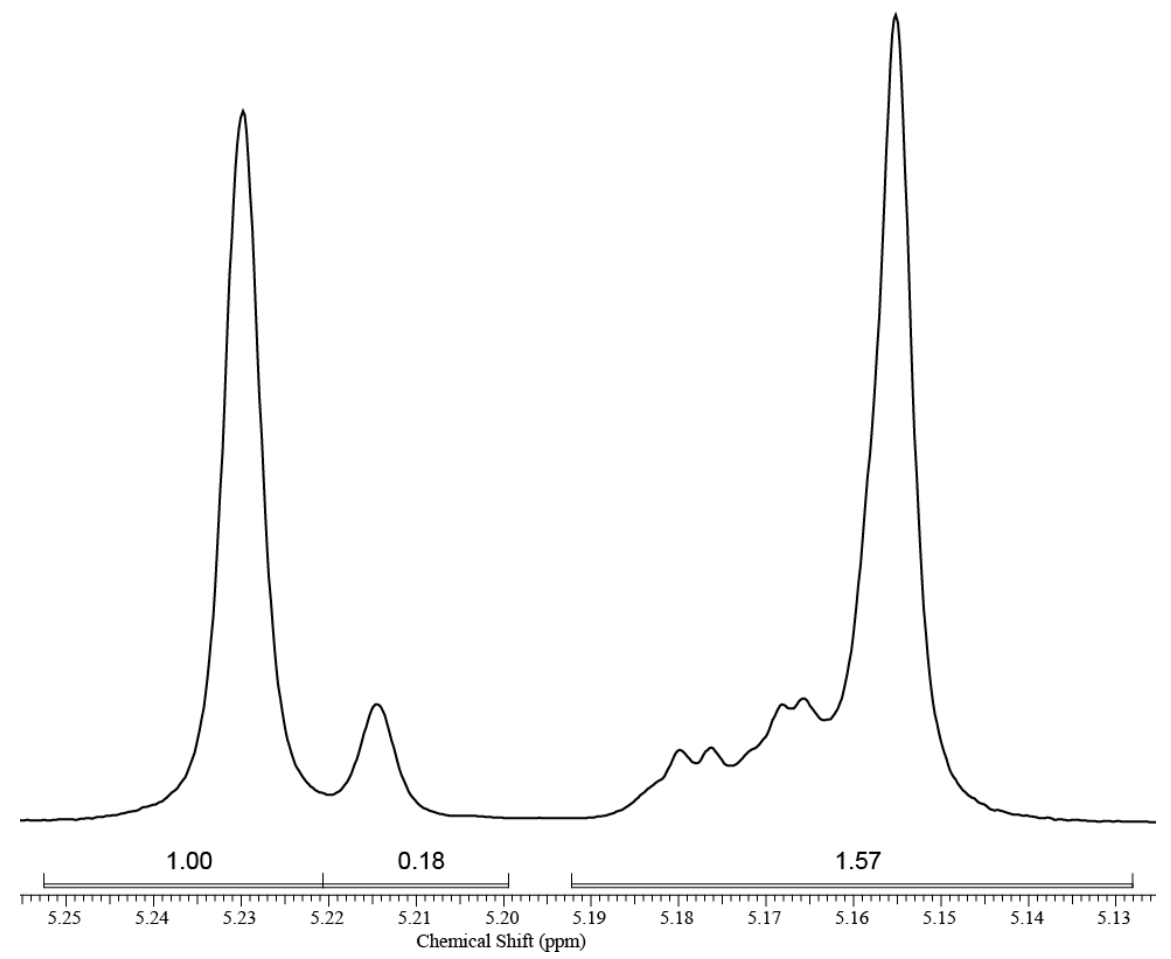

Figure S43. Homonuclear decoupled ${ }^{1} \mathrm{H}$ NMR spectrum of methine region of PLA prepared by complex $\mathbf{5}$ at lower temperatures $\left(\mathrm{P}_{\mathrm{r}}=0.85\right)$. 


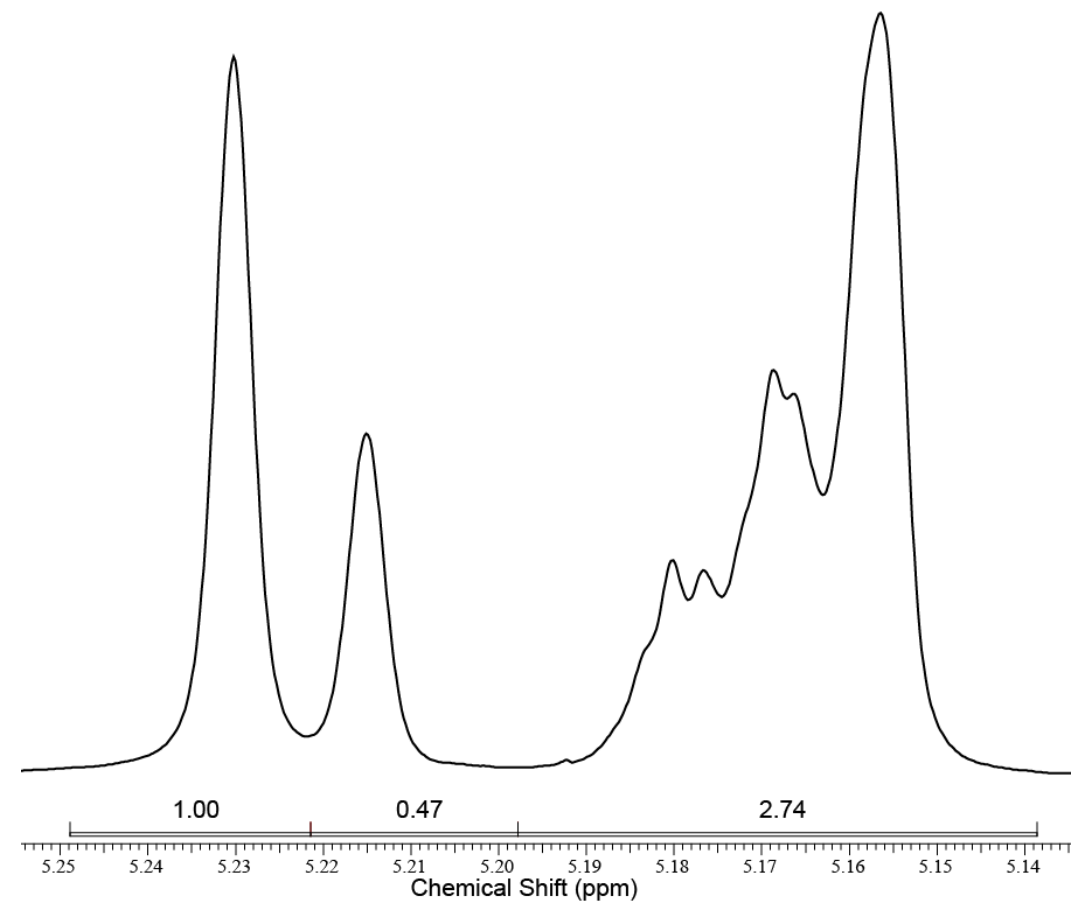

Figure S44. Homonuclear decoupled ${ }^{1} \mathrm{H}$ NMR spectrum of methine region of PLA prepared by complex $6\left(\mathrm{P}_{\mathrm{r}}=\right.$ $0.7)$. 


\section{I. ${ }^{1}$ H NMR spectra of PLLA formed by complexes 4-6}

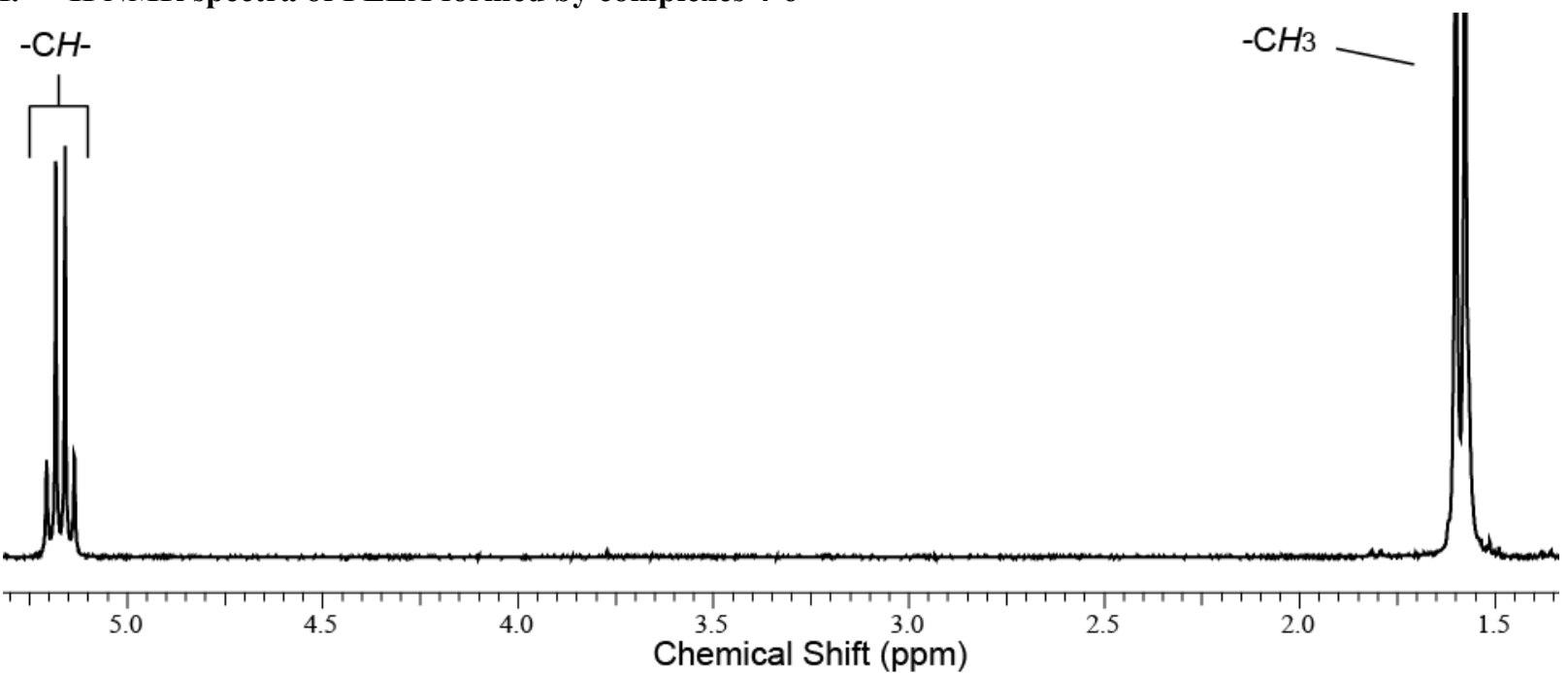

Figure S45. ${ }^{1} \mathrm{H}$ NMR spectrum of PLLA formed by complex $4\left(300 \mathrm{MHz}, 25^{\circ} \mathrm{C}, \mathrm{CDCl}_{3}\right)$.

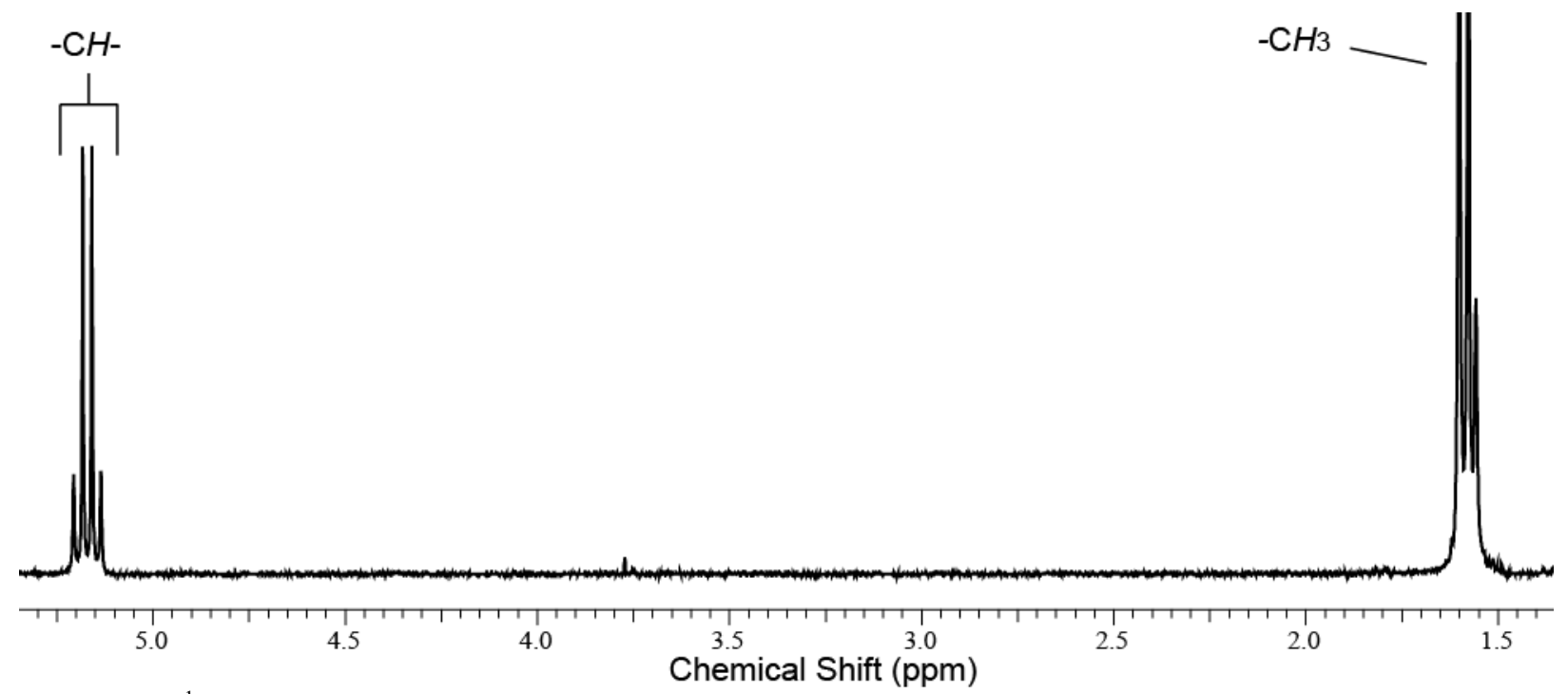

Figure S46. ${ }^{1} \mathrm{H}$ NMR spectrum of PLLA formed by complex $5\left(300 \mathrm{MHz}, 25{ }^{\circ} \mathrm{C}, \mathrm{CDCl}_{3}\right)$. 


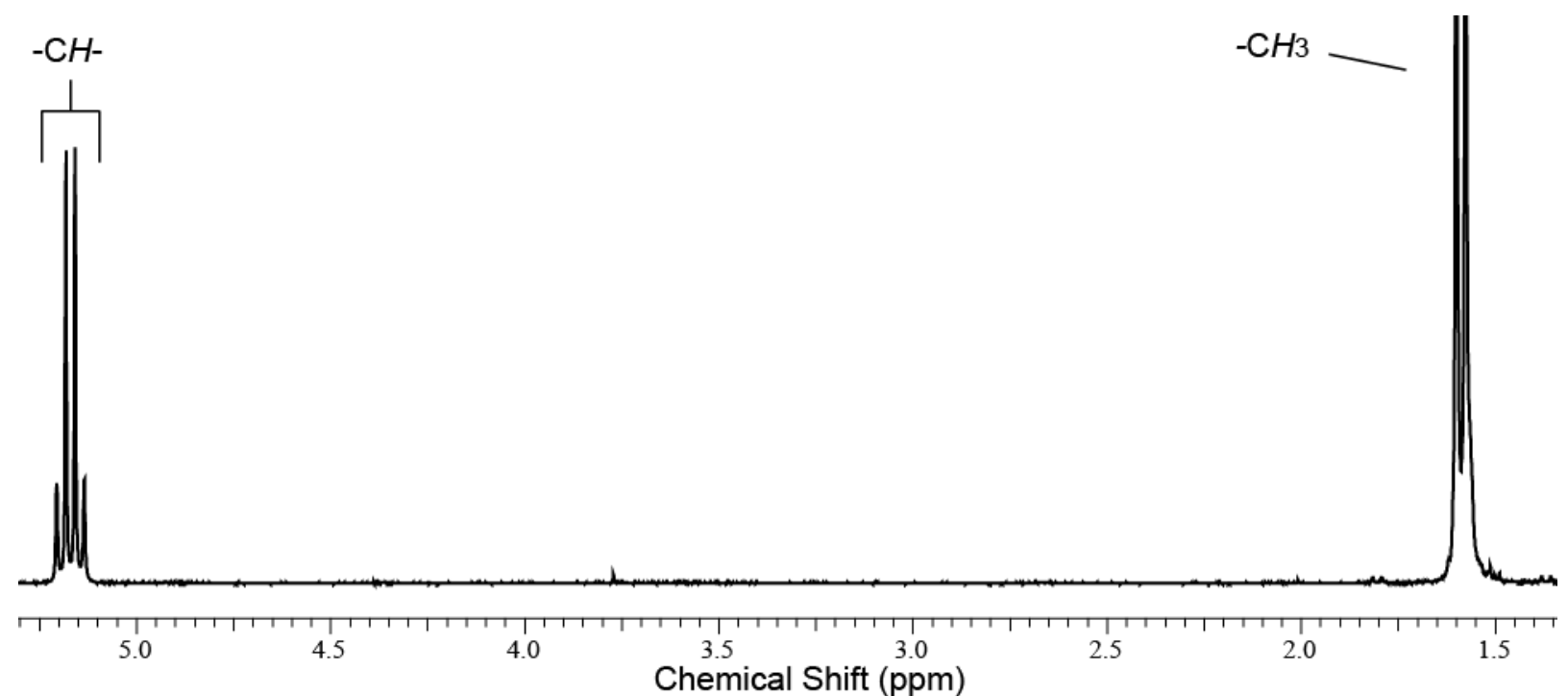

Figure S47. ${ }^{1} \mathrm{H}$ NMR spectrum of PLLA formed by complex $6\left(300 \mathrm{MHz}, 2{ }^{\circ} \mathrm{C}, \mathrm{CDCl}_{3}\right)$.

\section{J. References}

1. (a) Chamberlain, B. M.; Cheng, M.; Moore, D. R.; Ovitt, T. M.; Lobkovsky, E. B.; Coates, G. W., J. Am. Chem. Soc. 2001, 123 (14), 3229-3238; (b) Bovey, F. A.; Mirau, P. A. NMR of Polymers; Academic Press, San Diego, 1996.

2. Thakur, K. A. M.; Kean, R. T.; Zell, M. T., Padden, B. E.; Munson, E. J., Chem. Commun. 1998, (17), 1993-1994. 